ACCEPTED FOR PUBliCATION IN ApJ ON 08/05/2017

Preprint typeset using $\mathrm{LAT}_{\mathrm{E}} \mathrm{X}$ style AASTeX6 v. 1.0

\title{
THE INFRARED AND RADIO FLUX DENSITIES OF GALACTIC HII REGIONS
}

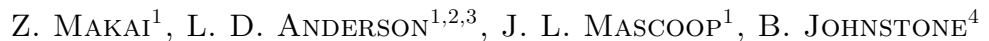 \\ ${ }^{1}$ Department of Physics and Astronomy, West Virginia University, Morgantown WV 26506 \\ ${ }^{2}$ Adjunct Astronomer at the Green Bank Observatory, P.O. Box 2, Green Bank WV 24944 \\ ${ }^{3}$ Center for Gravitational Waves and Cosmology, West Virginia University, Chestnut Ridge Research Building, Morgantown, WV 26505 \\ ${ }^{4}$ Benjamin M. Statler College of Engineering and Mineral Resources, West Virginia University, Morgantown, WV 26506
}

\section{ABSTRACT}

We derive infrared and radio flux densities of all $~ 1000$ known Galactic H II regions in the Galactic longitude range $17.5<\ell<65^{\circ}$. Our sample comes from the Wide-Field Infrared Survey Explorer (WISE) catalog of Galactic H II regions (Anderson et al. 2014). We compute flux densities at six wavelengths in the infrared (Spitzer GLIMPSE $8 \mu \mathrm{m}$, WISE $12 \mu \mathrm{m}$ and $22 \mu \mathrm{m}$, Spitzer MIPSGAL $24 \mu \mathrm{m}$, and Herschel Hi-GAL $70 \mu \mathrm{m}$ and $160 \mu \mathrm{m}$ ) and two in the radio (MAGPIS $20 \mathrm{~cm}$ and VGPS $21 \mathrm{~cm}$ ). All H II region infrared flux densities are strongly correlated with their $\sim 20 \mathrm{~cm}$ flux densities. All H II regions used here, regardless of physical size or Galactocentric radius, have similar infrared to radio flux density ratios and similar infrared colors, although the smallest regions $(r<1 \mathrm{pc})$, have slightly elevated IR to radio ratios. The colors $\log _{10}\left(F_{24 \mu \mathrm{m}} / F_{12 \mu \mathrm{m}}\right) \geq 0$ and $\log _{10}\left(F_{70 \mu \mathrm{m}} / F_{12 \mu \mathrm{m}}\right) \geq 1.2$, and $\log _{10}\left(F_{24 \mu \mathrm{m}} / F_{12 \mu \mathrm{m}}\right) \geq 0$ and $\log _{10}\left(F_{160 \mu \mathrm{m}} / F_{70 \mu \mathrm{m}}\right) \leq 0.67$ reliably select H II regions, independent of size. The infrared colors of $\sim 22 \%$ of H II regions, spanning a large range of physical sizes, satisfy the IRAS color criteria of Wood \& Churchwell (1989a) for HII regions, after adjusting the criteria to the wavelengths used here. Since these color criteria are commonly thought to select only ultra-compact $\mathrm{H}$ II regions, this result indicates that the true ultra-compact $\mathrm{H}$ II region population is uncertain. Comparing with a sample of IR color indices from star-forming galaxies, $\mathrm{H}$ II regions show higher $\log _{10}\left(F_{70 \mu \mathrm{m}} / F_{12 \mu \mathrm{m}}\right)$ ratios. We find a weak trend of decreasing infrared to $\sim 20 \mathrm{~cm}$ flux density ratios with increasing $R_{\text {gal }}$, in agreement with previous extragalactic results, possibly indicating a decreased dust abundance in the outer Galaxy.

Keywords: H II regions - infrared: ISM - radio continuum: ISM - techniques: photometric

\section{INTRODUCTION}

High-mass stars form in dense molecular clouds located primarily in spiral arms. Gas in the vicinity of these stars can be ionized by ultra-violet photons from the high-mass stars, creating $\mathrm{H}$ II regions. Because these high mass stars are short-lived, H II regions trace star formation in the current epoch. The radio and infrared (IR) emission from HII regions is bright, allowing the study of star formation across the entire Galaxy. H II regions can therefore be used to study global galactic properties.

The IR emission associated with $\mathrm{H}$ II regions comes from dust, and much of this dust is located in their photodissociation regions (PDRs) (Harper 1974). The PDR is the boundary between the ionized gas of the $\mathrm{H}$ II region itself and the ambient interstellar medium. At the wavelengths used here, 8.0, 12, 22, 24, 70 and $160 \mu \mathrm{m}$, the IR emission is due to a variety of different dust populations, as explained by (Robitaille et al. 2012). At 8.0 and $12 \mu \mathrm{m}$, dust emission is dominated by fluorescently excited polycyclic aromatic hydrocarbons (PAHs), which also contribute to the emission in the 22 and $24 \mu \mathrm{m}$ bands. Small grains emit in the 22 and $24 \mu \mathrm{m}$ bands, and also contribute substantially to the emission at $70 \mu \mathrm{m}$. Large grains contribute to the $70 \mu \mathrm{m}$ emission, but dominate the emission at $160 \mu \mathrm{m}$. Although emission in all these photometric bands is generically due to dust, each band is sensitive to different dust populations excited by different mechanisms. Also, the size and temperature of grains influence the observed IR spectral energy distribution (SED), i.e. more small grains can produce more $24 \mu \mathrm{m}$ emission that can shift the SED peak to the shorter wavelengths.

IR photometry of $\mathrm{HII}$ regions began $\sim 40$ years ago, with far-infrared (FIR) observations of the Orion and Omega nebulae (Low \& Aumann 1970). More recently, Phillips \& Ramos-Larios (2008) performed IR photometry of $58 \mathrm{H}$ II regions from $3.6 \mu \mathrm{m}$ to $8.0 \mu \mathrm{m}$, using Spitzer Galactic Legacy Infrared Mid-Plane Survey Extraordinaire (GLIMPSE; Churchwell \& GLIMPSE Team 2001; 
Benjamin et al. 2003) data. They found that the PDRs associated with H II regions are bright at mid-IR wavelengths. Previous studies also found that IR flux density ratios, or "colors," can be used to separate H II regions from other objects. Wood \& Churchwell (1989a, hereafter WC89) reported that ultra compact (UC) H II regions have IR colors of $\log _{10}\left(F_{60 \mu \mathrm{m}} / F_{12 \mu \mathrm{m}}\right)>1.30$ and $\log _{10}\left(F_{25 \mu \mathrm{m}} / F_{12 \mu \mathrm{m}}\right)>0.57$, where $F_{\lambda}$ denotes the flux density value at wavelength $\lambda$. Infrared flux density ratios can also be used to separate H II regions from planetary nebulae (PNe; Anderson et al. 2012). IR colors can also be used to characterize the star formation rate of entire galaxies (e.g. Temi et al. 2009).

H II regions in our Galaxy help us to better understand the IR radiation from galaxies in the local Universe. For example, IR flux density ratios can be used to investigate the star-formation and dust properties of local galaxies (e.g. Helou 1986; Soifer \& Neugebauer 1991; Wang 1991; Sanders et al. 2003). These and similar studies have found that the observed mid- and far-IR emission from galaxies contain contributions from warm and cooler dust components, and that this emission can be used to determine the star formation rate of galaxies.

Radio continuum emission from $\mathrm{H}$ II regions is due to (thermal) Bremsstrahlung radiation. Early radio continuum observations found that most bright radio continuum sources are Galactic H II regions (see e.g. Piddington 1951; Haddock et al. 1954; Westerhout 1958). Harper (1974, and references therein) noticed a linear correlation between $\mathrm{H}$ II region far-IR and radio $(2 \mathrm{~cm})$ flux densities. A strong correlation between IR emission (from "warm" dust emitting at $60 \mu \mathrm{m}$ ) and radio continuum emission (at $11 \mathrm{~cm}$ ) for $\mathrm{H}$ II regions was also reported in Haslam \& Osborne (1987) and Broadbent et al. (1989).

Infrared and radio flux densities are also strongly correlated on galactic scales. de Jong et al. (1985) showed a strong linear correlation between $6.3 \mathrm{~cm}$ radio continuum and $60 \mu \mathrm{m}$ far-infrared flux densities for a sample of spiral, irregular, and dwarf galaxies. The radio continuum emission at $6.3 \mathrm{~cm}$ is due to thermal emission associated with high-mass star formation and non-thermal synchrotron emission. The $60 \mu \mathrm{m}$ emission is from warm dust associated with star formation. The correlation indicates that both radio continuum and far-infrared emission trace star formation activity. This has also been shown by e.g. Tabatabaei et al. (2013) and by Mingo et al. (2016, and references therein).

Here, we examine the infrared and radio flux densities of a large sample of inner-Galaxy H II regions, determine relationships between these flux densities, and search for variations with H II region radius and Galactocentric radius. In Section 2, we describe the H II region sample and the data sets used. We explain our aperture pho- tometry methodology in Section 3, and examine correlations between the IR and radio flux densities in Section 4. The summary follows in Section 5.

\section{DATA}

\subsection{Source Selection}

The WISE Catalog of Galactic H II Regions contains all known and candidate H II regions in the Galaxy (Anderson et al. 2014). We use catalog Version 1.5, available at http://www.astro.phys.wvu.edu/wise. The cata$\log$ lists $\sim 1900$ known H II regions that have measured ionized gas spectroscopic lines ( $\mathrm{H} \alpha$ or radio recombination lines). There are an additional 700 "grouped" $\mathrm{H}$ II regions that are part of large star-forming complexes like W49 or W51, but which have not been individually targeted for ionized gas spectroscopic observations. We here use both the known and group HII regions. The remaining $\sim 5800$ catalog entries are H II region candidates that we do not consider further. We restrict the $\mathrm{H}$ II region sample to the Galactic longitude range of $17.5<\ell<65^{\circ}$ and, because our photometric data are limited in Galactic latitude, to $|b|<2.5$. Our final sample of known and group H II regions contains 1011 sources.

In the longitude and latitude zone of the present work, the WISE catalog lists Heliocentric distances for 525 known H II regions and 85 group regions. It lists Galactocentric distances for 717 known regions and 126 group regions. The group HII region distances come exclusively from molecular line experiments, and not from their association with the known regions (see Anderson et al. 2014). Most of the catalog distances are kinematic. Over the longitude range used here, kinematic distances are relatively accurate, assuming the kinematic distance ambiguity is correctly resolved (T.V. Wenger et al., 2017, in prep.). Also, due to recent HiI region surveys (e.g. Anderson et al. 2011, 2014), the sample is by a large margin more complete here than in the rest of the Galaxy.

\subsection{GLIMPSE}

GLIMPSE is a Spitzer legacy survey of the inner Galactic plane, covering $-65^{\circ} \lesssim \ell \lesssim 65^{\circ},|b| \lesssim 1^{\circ}$. The data were taken with the Infrared Array Camera (IRAC, Fazio et al. 1998) in four different infrared bands $(3.6 \mu \mathrm{m}, 4.5 \mu \mathrm{m}, 5.8 \mu \mathrm{m}$ and $8.0 \mu \mathrm{m})$ at resolutions of $\sim 2^{\prime \prime}$. These emission bands contain strong PAH features at $3.3 \mu \mathrm{m}, 6.2 \mu \mathrm{m}, 7.7 \mu \mathrm{m}$ and $8.6 \mu \mathrm{m}$, and many weaker PAH "plateaus" at slightly longer wavelengths (Andrews et al. 2015). Here we use only the $8.0 \mu \mathrm{m}$ data, which for $\mathrm{H}$ II regions is dominated by $\mathrm{PAH}$ emission.

Scattering within the focal plane causes higher measured flux densities of extended sources with the IRAC 
instrument. To correct this effect (which is wavelength dependent), we follow the Spitzer recommendations ${ }^{1}$ and apply an aperture correction to the $8.0 \mu \mathrm{m}$ flux densities, based on the aperture size. This correction lowers the measured flux densities values by a maximum of $35 \%$ for an aperture of 50". Following the Spitzer instrument handbook recommendation ${ }^{2}$, we did not apply a color correction factor to the GLIMPSE flux densities.

\subsection{WISE}

The Wide-field Infrared Survey Explorer (WISE; Wright et al. 2010) mapped the entire sky at four wavelengths: $3.4 \mu \mathrm{m}, 4.6 \mu \mathrm{m}, 12 \mu \mathrm{m}$, and $22 \mu \mathrm{m}$. The angular resolutions are $6.1^{\prime \prime}, 6.4^{\prime \prime}, 6.5^{\prime \prime}$ and $12^{\prime \prime}$ with the $5 \sigma$ sensitivities of $0.08 \mathrm{mJy}, 0.11 \mathrm{mJy}, 1 \mathrm{mJy}$ and $6 \mathrm{mJy}$, respectively. We use also here the $12 \mu \mathrm{m}$ and $22 \mu \mathrm{m}$ bands (which we also refer to as $\mathrm{W} 3$ and $\mathrm{W} 4$, respectively). The $12 \mu \mathrm{m}$ emission mechanism is similar to that of the $8.0 \mu \mathrm{m}$ GLIMPSE data, in that it is also sensitive to $\mathrm{PAH}$ features, at $11.2 \mu \mathrm{m}, 12.7 \mu \mathrm{m}$ and $16.4 \mu \mathrm{m}$ (e.g. Roser \& Ricca 2015; Tielens 2008). As the WISE data have DN units, we used the DN-to-Jy conversion factors of $2.9045 \times 10^{-6}$ and $5.2269 \times 10^{-6}$ in cases of $12 \mu \mathrm{m}$ and $22 \mu \mathrm{m}$, respectively ${ }^{3}$. We use the color-corrections of Wright et al. (2010), assuming a spectral index of $\alpha=0$. This correction raises the W3 flux densities by $9.1 \%$ and the W4 flux densities by $1.0 \%$.

The W4 $22 \mu \mathrm{m}$ bandpass is similar to that of the Spitzer MIPS instrument used for the $24 \mu \mathrm{m}$ MIPSGAL survey described below. Both $\sim 20 \mu \mathrm{m}$ data are sensitive to stochastically-heated very small grains (VSGs) within the $\mathrm{H}$ II region plasma, and also to dust grains within the PDRs (PAHs are prominant contributors of $24 \mu \mathrm{m}$ emission, Robitaille et al. (2012)). Deharveng et al. (2010) showed that roughly half of the dust emission traced by the MIPSGAL $24 \mu \mathrm{m}$ emission originates from the interior of $\mathrm{H}$ II regions.

\subsection{MIPSGAL}

MIPSGAL is a Spitzer Galactic plane survey using the Multiband Infrared Photometer for Spitzer (MIPS; Rieke et al. 2004) photometer (Carey et al. 2005). Like GLIMPSE, it covers $-65^{\circ} \lesssim \ell \lesssim 65^{\circ},|b| \lesssim 1^{\circ}$. We use the $24 \mu \mathrm{m}$ MIPSGAL data here, which has a resolution of $6^{\prime \prime}$. MIPSGAL saturates at $1700 \mathrm{MJy} \mathrm{sr}^{-1}$ in cases of extended sources at $24 \mu \mathrm{m}$ and, contrary to the IRAC bands, MIPSGAL flux densities have a negligible

\footnotetext{
1 http://irsa.ipac.caltech.edu/data/SPITZER/docs/irac/ iracinstrumenthandbook/29/\$\#\$_Toc410728320

2 http://irsa.ipac.caltech.edu/data/SPITZER/docs/irac/ iracinstrumenthandbook/18/\$\#_Toc410728306

3 For more information, see http://wise2.ipac.caltech.edu/ docs/release/prelim/expsup/wise_prelrel_toc.html
}

correction factor for scattering in the focal plane (Cohen 2009). We use the (small) color correction factor given in the MIPS Instrument Handbook ${ }^{4}$. This correction is appropriate for 100 to $1000 \mathrm{~K}$ dust and raises the MIPSGAL flux densities by $3.5 \%$.

\section{5. $H i-G A L$}

The Herschel infrared Galactic Plane Survey (HiGAL; Molinari et al. 2010, 2016) used the PACS (Poglitsch et al. 2010) and SPIRE (Griffin et al. 2010) instruments on board the Herschel Space Observatory (Pilbratt et al. 2010) to map the entire Galactic plane within $|b| \leq 1^{\circ}$. The photometric bands are centered at $70 \mu \mathrm{m}, 160 \mu \mathrm{m}$ (PACS), and $250 \mu \mathrm{m}, 350 \mu \mathrm{m}$ and $500 \mu \mathrm{m}$ (SPIRE). We use here only the $70 \mu \mathrm{m}$ and $160 \mu \mathrm{m}$ PACS data. Although the dust associated with $\mathrm{H}$ II regions do emit in the longer-wavelength data (Anderson et al. 2012), at these wavelengths the associated emission is difficult to disentangle from the background. The IRAS $60 \mu \mathrm{m}$ and the PACS $70 \mu \mathrm{m}$ bands trace emission from the same dust components, and include contributions from both very small grains and large grains (Paladini et al. 2012). At $160 \mu \mathrm{m}$ the emission is almost entirely due to the large grains. The Hi-GAL point source sensitivities are $0.5 \mathrm{Jy}_{\text {beam }}{ }^{-1}$ and $4.1 \mathrm{Jy} \mathrm{beam}^{-1}$ in complex fields and the spatial resolutions are $6 .{ }^{\prime \prime} 7$ and $11^{\prime \prime}$, in the $70 \mu \mathrm{m}$ and $160 \mu \mathrm{m}$ bands, respectively (Molinari et al. 2010). We applied the color corrections given in the PACS Photometer-Colour Corrections document ${ }^{5}$. Our correction is appropriate for 30 to $100 \mathrm{~K}$ dust and increases the $70 \mu \mathrm{m}$ and $160 \mu \mathrm{m}$ flux densities by $1 \%$.

\subsection{MAGPIS}

The Multi-Array Galactic Plane Imaging Survey (MAGPIS) $20 \mathrm{~cm}$ data (Helfand et al. 2006) covers a portion of the first Galactic quadrant $\left(5^{\circ}<\ell<48.5\right.$, $|b|<0.8)$. These data were created using multiple VLA configurations in addition to Effelsburg single-dish data, and so are sensitive to a range of spatial scales. The MAGPIS point source detection threshold is $\sim 2 \mathrm{mJy}$, excluding bright extended emissions, and the angular resolution is $\sim 6^{\prime \prime}$. As the MAGPIS data possess higher angular resolution than the VGPS data $\left(\sim 6^{\prime \prime}\right.$ vs. $1^{\prime}$; see below), the MAGPIS data can be used to more accurately separate the emission from compact $\mathrm{H}$ II regions from that of the background.

Helfand et al. (2006) reported a possible inaccurate flux density scale for large sources. They compared the

\footnotetext{
4 http://irsa.ipac.caltech.edu/data/SPITZER/docs/mips/ mipsinstrumenthandbook/51/\#_Toc288032329

5 Website: http://herschel.esac.esa.int/twiki/bin/view/ Public/PacsCalibrationWeb
} 
MAGPIS flux densities of 25 known SNRs with values from the literature compiled by Green (2004). They found a reasonably good correlation between the flux density values, but with overestimation of the true flux density by a factor of 2 due to the backgrounds, and contaminating sources (some possible reasons are given in Figure 6 of Helfand et al. 2006).

\section{7. $V G P S$}

We also use radio continuum data from the Very Large Array (VLA) Galactic Plane Survey (VGPS), which mapped the $21 \mathrm{~cm}$ emission from neutral atomic hydrogen (HI). The VGPS survey covers $18^{\circ}<\ell<$ $67^{\circ},|b|<2.5$ with $1^{\prime}$ resolution and a sensitivity of 11 mJy beam $^{-1}$ (Stil et al. 2006). In addition to the H I data, the VGPS produced the radio continuum data used here using line-free portions of the spectra. They filled in the continuum zero-spacing using the Effelsberg data from Reich \& Reich (1986) and Reich et al. (1990).

\section{8. $H R D S$}

We also use $3 \mathrm{~cm}$ radio continuum data from the Green Bank Telescope (GBT) H II Region Discovery Survey (HRDS; Bania et al. 2010). The original HRDS covers $-17^{\circ}<\ell<67^{\circ},|b|<1^{\circ}$ (Anderson et al. 2011). The HRDS extension (Anderson et al. 2014) covered the entire sky north of a declination of $-45^{\circ}$, which is equivalent to $-20^{\circ} \leqslant \ell \leqslant 270^{\circ}$ at $b=0^{\circ}$. The HRDS continuum was created using total-power cross scans in RA and Dec for each source. For the current twork, we only use sources from HRDS whose continuum emission profile could be modeled by a single Gaussian, and whose peak emission as derived from the cross scans was within $10^{\prime \prime}$ of the targeted position.

\section{APERTURE PHOTOMETRY}

Radio continuum emission traces the ionized gas content of an Hil region. Mid- to far-infrared emission traces dust that is co-spatial with the ionized gas (notably the $\sim 20 \mu \mathrm{m}$ and $70 \mu \mathrm{m}$ emission from very small grains), and also from the PDR (the $\sim 10 \mu \mathrm{m}$ and $160 \mu \mathrm{m}$ emission from PAHs and large grains, respectively). Hereafter, we use language suggesting that the radio continuum and MIR emission are both from the H II region, although strictly speaking they come from different parts of the star formation region.

We perform aperture photometry using the Kang software $^{6}$. Since many H II regions in our sample are located close to objects that can contaminate the emission of the source and/or have irregular morphology, it is important to have a flexible aperture shape and size. When

\footnotetext{
${ }^{6}$ http://www.bu.edu/iar/kang/
}

computing aperture photometry, Kang apportions flux density from partial pixels, which can be important for smaller regions.

We define one source aperture and four background apertures for each source (see Figure 1). We use the angular extent of each region from the WISE catalog to guide our source aperture definitions. In many cases, we must remove contaminating point sources, which we do manually. We also remove any other contaminating HII regions that may fall within the source aperture. Our goal with the background apertures is to accurately sample the background in four cardinal directions, while avoiding extended emission from nearby sources. Our methodology is identical to that of Anderson et al. (2012).

Because HII regions have different sizes at infrared and radio wavelengths, and because the backgrounds differ between the infrared and radio, we define multiple sets of apertures. We define one set of source and background apertures for all the infrared data, one set for MAGPIS data, and one set for the VGPS data. Although the radio backgrounds should in principle be similar, the two radio aperture sets are required due to the very different spatial resolutions.

We perform aperture photometry using the equation

$$
S_{\nu}=S_{\nu, 0}-\frac{B_{\nu}}{N_{B}} \times N_{S},
$$

where $S_{\nu}$ is the source flux density (after background subtraction), $S_{\nu, 0}$ is flux density within the source aperture (without background subtraction), $B_{\nu}$ is the flux density in the background aperture, $N_{B}$ is the number of pixels within the background aperture, and $N_{S}$ is the pixel number within the source aperture. With this method, we subtract the mean flux density of the background aperture from every pixel in the source aperture. Because we have four background apertures, we have four values of $S_{\nu}$. We use in all subsequent analysis the mean of these four values, and take as the uncertainty their standard deviation.

\subsection{Handling uncertainties}

The choice of source aperture size is somewhat subjective, and results in additional photometric uncertainties. We attempt to quantify this uncertainty using the H II region G035.126 - 00.755 and GLIMPSE $8.0 \mu \mathrm{m}$ data. For this region, we compare the flux density derived using source apertures of four different sizes. We find that as the source aperture size increases, the backgroundsubtracted flux density also increases, with the largest apertures measuring flux densities nearly $20 \%$ higher than the smallest apertures. The uncertainties on the largest aperture flux densities are $\sim 30 \%$, whereas they are $\sim 10 \%$ for the smallest apertures. As the source 

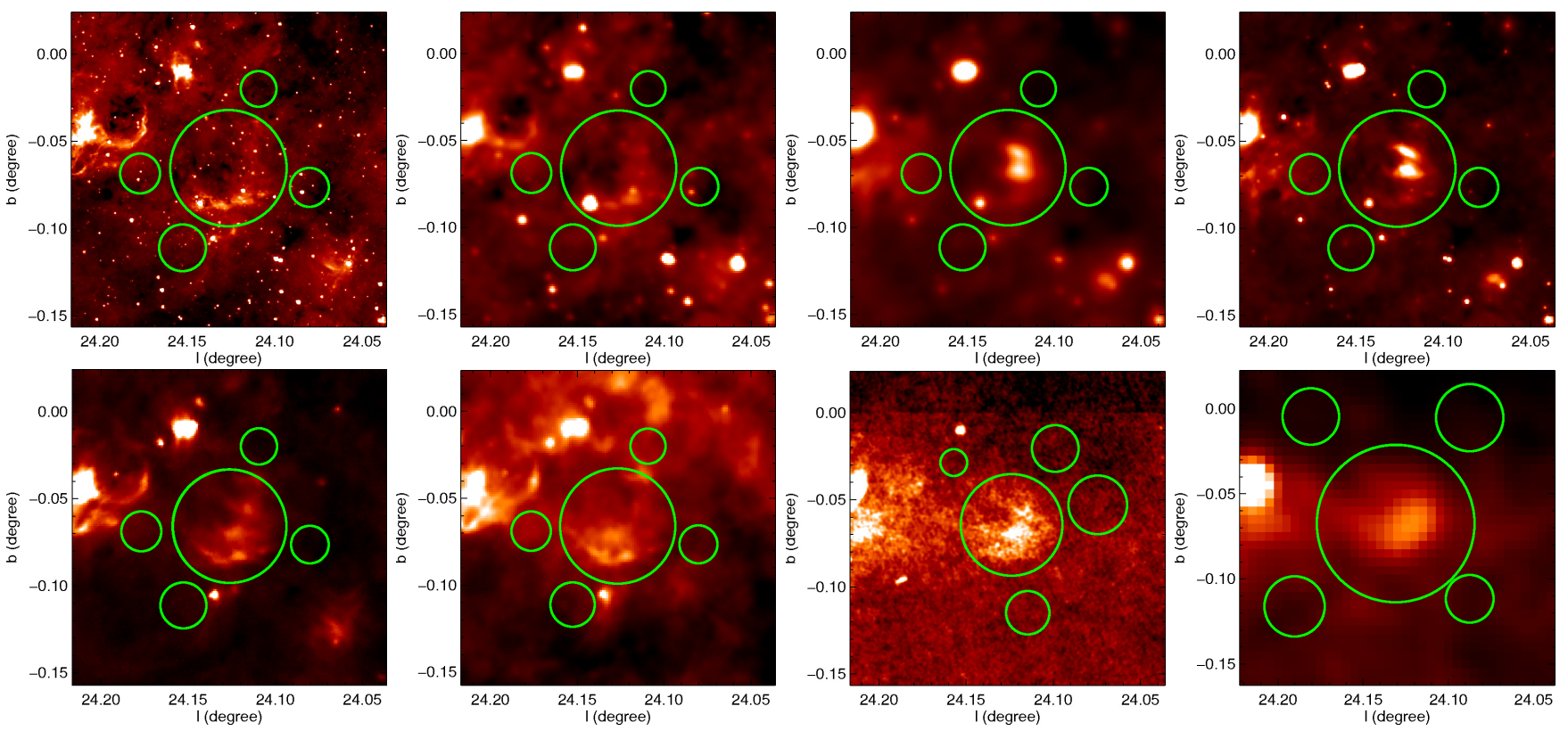

Figure 1. Example apertures for one HII region, G024.126-00.066. Top row, left to right: GLIMPSE $8.0 \mu \mathrm{m}, \mathrm{W} 312 \mu \mathrm{m}, \mathrm{W} 4$ $22 \mu \mathrm{m}$, and MIPSGAL $24 \mu \mathrm{m}$ data. Bottom row (from left to right): Hi-GAL $70 \mu \mathrm{m}$, Hi-GAL $160 \mu \mathrm{m}$, MAGPIS $20 \mathrm{~cm}$ and VGPS $21 \mathrm{~cm}$ data. The largest circle represents the source aperture, and the four smaller apertures are used for the background flux density derivation. The different background levels necessitate three different aperture sets, one for all IR data, one for MAGPIS, and one for the VGPS.

apertures increase in size, they sample more background emission, and are therefore more sensitive to the choice of background apertures. The GLIMPSE data have the strongest background variations, and other wavelengths may show a smaller effect. We conclude that the choice of source aperture size has an effect on the derived flux densities that is comparable to that of the photometric uncertainties derived from our four background apertures.

For clarity, we generally do not show photometric uncertainties in subsequent plots. We do show an analysis of the uncertainties in Appendix B. Based on this analysis, $50 \%$ of the data have $\lesssim 35 \%$ fractional uncertainties, while over $90 \%$ of the data have fractional uncertainties $\lesssim 200 \%$, at all wavelengths (see Figures B1, B2 and Table B1 in Appendix B).

There are a number of reasons why we cannot compute a flux density for a given souce at a given wavelength. Many sources are confused, and cannot be separated from nearby $\mathrm{H}$ II regions. In such cases, we do not compute aperture photometry measurements. Some sources are simply not detected at a given wavelength. The sky coverage for each survey is different and so excludes some sources.

We also exclude flux densities for sources that have more than $0.1 \%$ of all pixels that are so strongly saturated that they have a value of "NaN". This limit was found by Anderson et al. (2012) to be the best value when discriminating between sources whose flux densities were seriously impacted by saturation and those that were not. Due to saturation, we remove 18,21 , $58,71,2$ and 3 data points that correspond to $\sim 2.0 \%$, $\sim 2.3 \%, \sim 6.1 \%, \sim 7.5 \%, \sim 0.2 \%$ and $\sim 0.3 \%$ data loss from GLIMPSE $(8 \mu \mathrm{m})$, W3 $(12 \mu \mathrm{m})$, W4 $(22 \mu \mathrm{m})$, MIPSGAL $(24 \mu \mathrm{m})$ and Hi-GAL $(70 \mu \mathrm{m}$ and $160 \mu \mathrm{m})$ surveys, respectively. The radio continuum surveys (MAGPIS and VGPS) do not suffer from saturation.

\subsection{Source radius estimation}

The derivation of the size of an HII region is not straightforward because $\mathrm{H}$ II regions are not necessarily spherical, and because the H II region boundary can be difficult to define. We are interested in examining trends in $\mathrm{H}$ II region flux densities and flux density ratios as a function of $\mathrm{HII}$ region radius, and therefore we need a reliable method for estimating $\mathrm{H}$ II region radii.

We primarily use the MAGPIS radio apertures to estimate $\mathrm{H}$ II region radii. Since it has an angular resolution of $\sim 6^{\prime \prime}$ and traces the ionized gas, this is the most appropriate survey. Some larger diffuse regions are not detected in MAGPIS and therefore do not have MAGPIS apertures. For these, and for regions outside the range of the MAGPIS survey, we instead use the VGPS $21 \mathrm{~cm}$ apertures as long as the source has a diameter twice that of the VGPS resolution. For large regions, the MIR and radio continuum radii are similar. For the small number of regions with radii $>5^{\prime}$ where we are also missing MAGPIS and VGPS data, we use the infrared aperture radius. In total, we estimate $868 \mathrm{H} \mathrm{II}$ region radii; 609, 242 and 17 using the MAGPIS, VGPS 


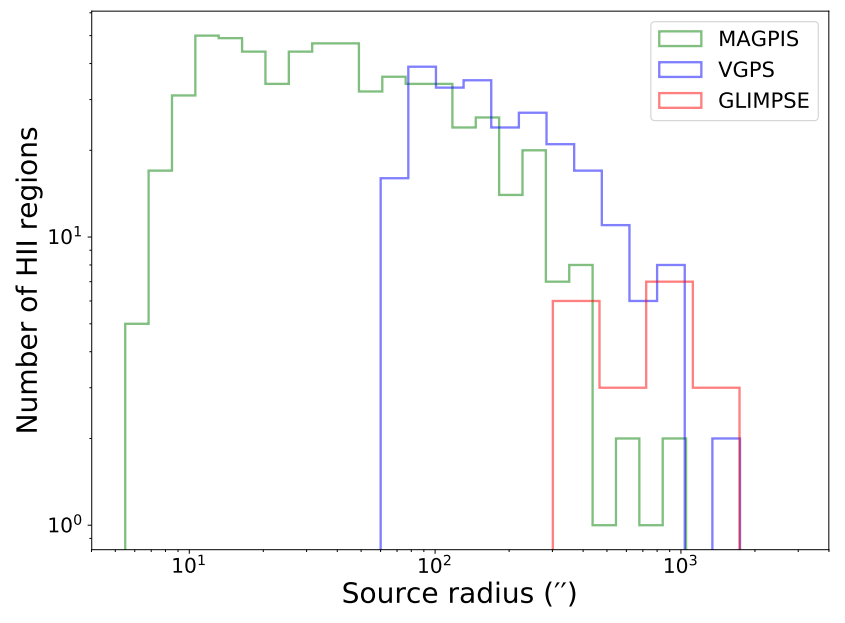

Figure 2. Distribution of the angular radii of H II regions. We estimate the radius of each $\mathrm{H}$ II region in the sample using MAGPIS (green), VGPS (blue), or IR (red) apertures (see Section 3.2). The radii of the smallest regions are mostly measured using MAGPIS data, whereas many of the largest regions are measured using GLIMPSE data.

and GLIMPSE apertures, respectively (Figure 2).

The radii we use in subsequent analyses are those corresponding to a circle of the same size as the aperture area (the apertures are not necessarily circular). In much of the following analysis, we use physical radii computed using the $\mathrm{H}$ II region distances compiled in the WISE catalog.

It is important to remember that these radii are only approximate, having been defined by-eye from radio continuum (and in some cases IR) data. Furthermore, the physical radii used later rely on accurate distances for the HII regions. The angular and physical radii are therefore not appropriate for detailed analyses of individual regions. We use these radii only statistically here.

\section{RESULTS}

We give the aperture photometry results in Table 1 , which includes the names, flux density values with their $1 \sigma$ uncertainties, angular radii, and physical radii.

We follow the same fitting method throughout the remainder of the paper. For each fit throughout the paper, we fit a power law of the form $F_{Y}=A F_{X}^{\alpha}$, where the terms $F_{X}$ and $F_{Y}$ denote the flux density values plotted on the $\mathrm{x}$ - and $\mathrm{y}$-axes. Each fit is determined using the least-squares method, and weights are computed using Orthogonal Distance Regression (ODR) method (Boggs \& Rogers 1990) that takes into account errors for both independent and dependent variables. Importantly, we perform the fit by taking the base-ten logarithm of the data and fitting a linear regression. This is equivalent to fitting the power law form above, but removes the bias toward the highest flux density values found when fitting the data itself using the least squares method. We compute the $R^{2}$ values as the goodness of fit statistic, using the linear fit and the base-ten logarithm of the data.

\subsection{IR flux density accuracy}

We compare our HiI region flux densities with those from the second version of the IRAS Point Source Catalog (PSC) to inspect the flux density calibrations and the aperture photometry methodology. We select isolated HII regions that can be found in the IRAS catalogs, and have measured IRAS flux densities at $12 \mu \mathrm{m}$, $25 \mu \mathrm{m}$, and $60 \mu \mathrm{m}$.

We compare our derived values with those from the IRAS PSC using the W3 $12 \mu \mathrm{m}$ versus IRAS $12 \mu \mathrm{m}$ data, the MIPSGAL $24 \mu \mathrm{m}$ versus IRAS $25 \mu \mathrm{m}$, and the HiGAL $70 \mu \mathrm{m}$ versus IRAS $60 \mu \mathrm{m}$ data (Figure 3). There is no systematic offset in any of these relationships, although there is large scatter. The standard deviation of the ratio of IRAS PSC $F_{12 \mu \mathrm{m}}$ and W3 $F_{12 \mu \mathrm{m}}$ is $\sim 58 \%$ from unity. In case of the IRAS PSC $F_{25} \mu \mathrm{m}$ and MIPSGAL $F_{24 \mu \mathrm{m}}$ ratio, the spread of the data points is larger, with a standard deviation of $\sim 81 \%$. The ratio of IRAS PSC $F_{60 \mu \mathrm{m}}$ and Hi-GAL $F_{70 \mu \mathrm{m}}$ has a standard deviation of $\sim 51 \%$.

GLIMPSE $8.0 \mu \mathrm{m}$ and W3 $12 \mu \mathrm{m}$ flux densities, as well as MIPSGAL $8.0 \mu \mathrm{m}$ and W4 $22 \mu \mathrm{m}$ flux densities, are strongly correlated (Figure 4): the slopes of the fits are $0.964 \pm 0.005$ and $1.070 \pm 0.006$ for GLIMPSE versus W3 and MIPSGAL versus W4, respectively. Since both the GLIMPSE and W3 data trace PAH emission, the strong linear correlation is unsurprising. The W4 and MIPSGAL bandpasses are similar, and therefore the strong correlation between these quantities is also unsurprising. The standard deviations from the unity line ratio are $\sim 60 \%$ and $\sim 34 \%$ for the W3 $(12 \mu \mathrm{m}) /$ GLIMPSE $(8 \mu \mathrm{m})$ ratio and $\mathrm{W} 4(22 \mu \mathrm{m}) /$ MIPSGAL $(24 \mu \mathrm{m})$ ratio, respectively.

\subsection{Radio flux density accuracy}

Since the VGPS and MAGPIS surveys are at approximately the same wavelength, their flux densities should also be highly correlated. Helfand et al. (2006), however, reported a possible large-scale calibration problem for the MAGPIS data. They computed the MAGPIS flux densities for 25 known SNRs and compared those values with those given in Green (2004). They found that MAGPIS overestimates flux densities, but that the discrepancy is reduced by subtracting $0.07 \mathrm{Jy} \operatorname{arcmin}^{-2}$.

Contrary to Helfand et al. (2006), we find that the MAGPIS and VGPS flux density values do not have a significant offset (Figure 5). This result also holds independently for large $\mathrm{H}$ II regions $>6^{\prime}$ in radius, orangered dots on Figure 5. In the sample of SNRs used in Helfand et al. (2006), there are 16 SNRs that have radii 


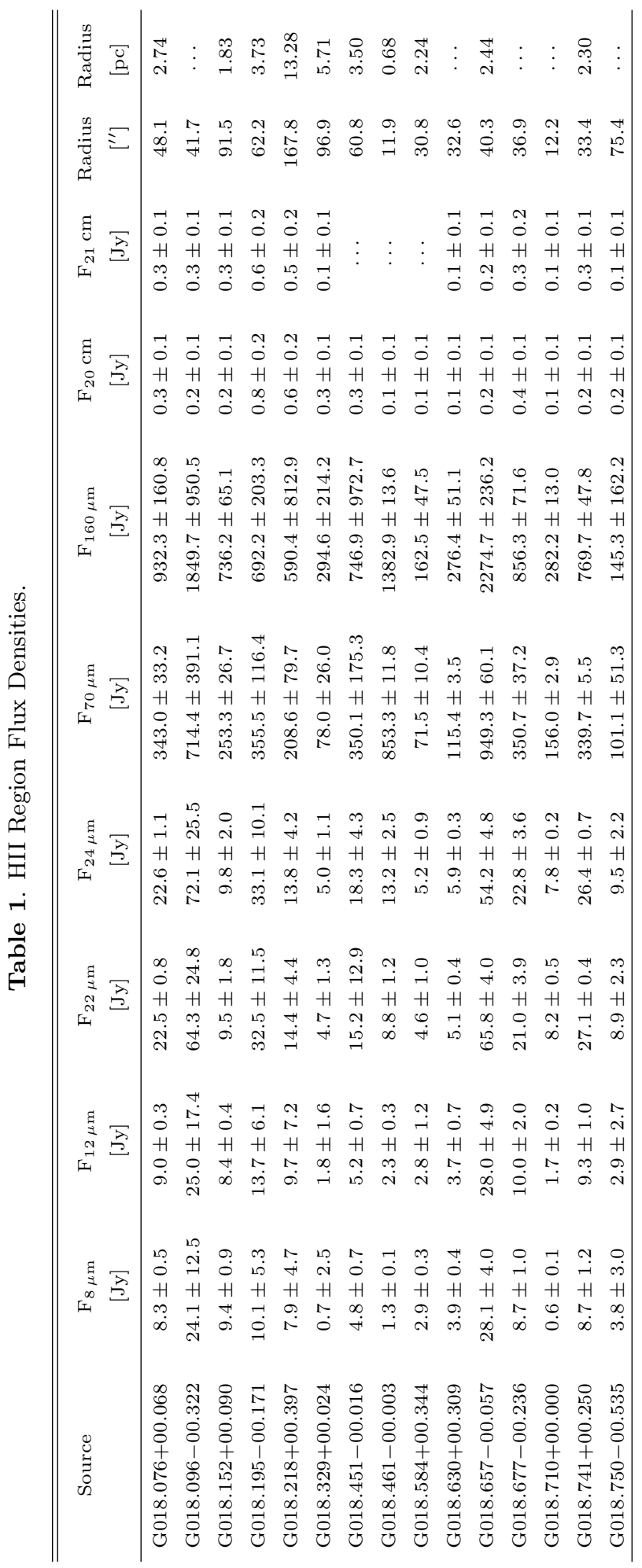



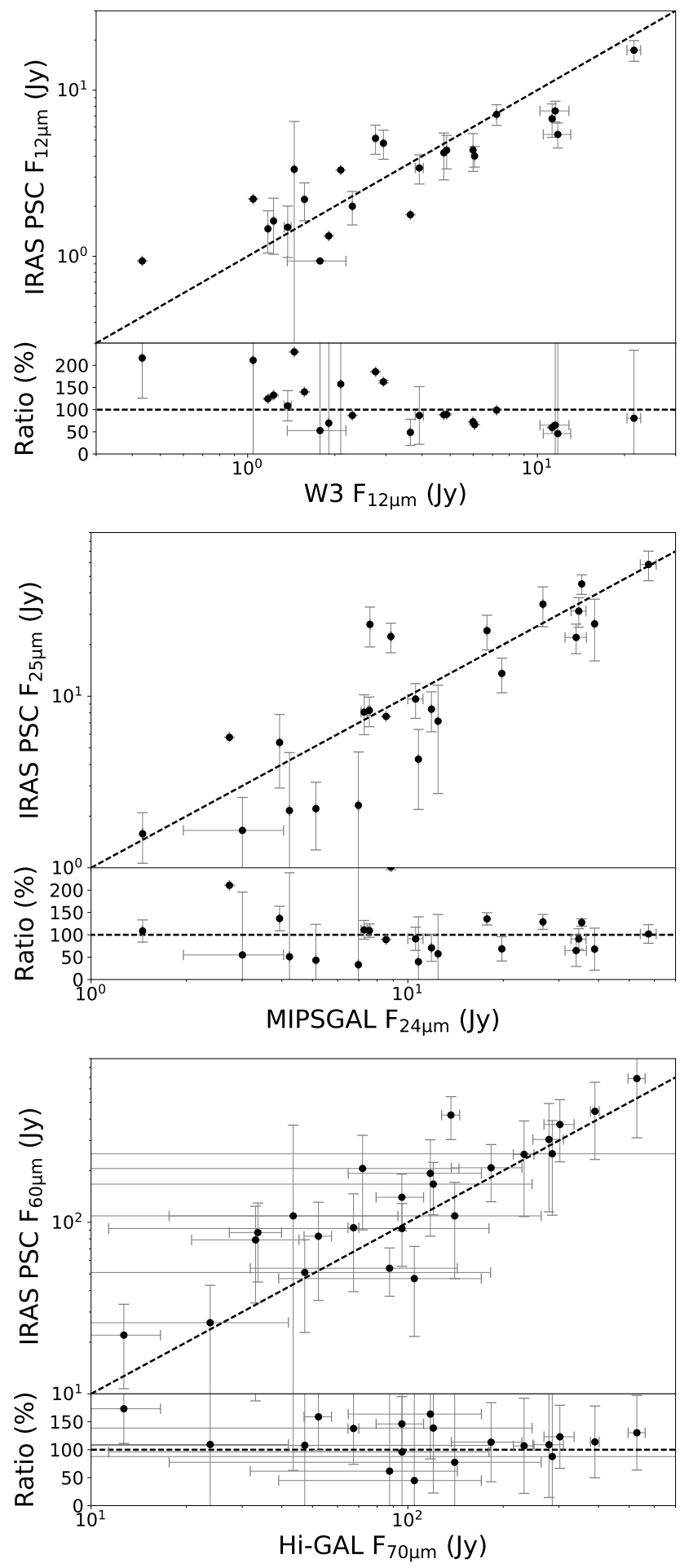

Figure 3. Comparison of IRAS PSC $(12 \mu \mathrm{m})$ and W3 fluxes densities (top subplot), IRAS PSC $25 \mu \mathrm{m}$ and MIPSGAL $(24 \mu \mathrm{m})$ flux densities (middle subplot), and IRAS PSC $60 \mu \mathrm{m}$ and Hi-GAL $(70 \mu \mathrm{m})$ flux densities (bottom subplot). The black dashed lines mark the 1:1 ratio. The lower panels show the deviation from the 1:1 ratio. The $3 \sigma$ photometric uncertainties are represented by the errorbars. The various flux densities are strongly correlated, with no obvious systematic offset. The significant scatter is likely due to the photometric uncertainties.
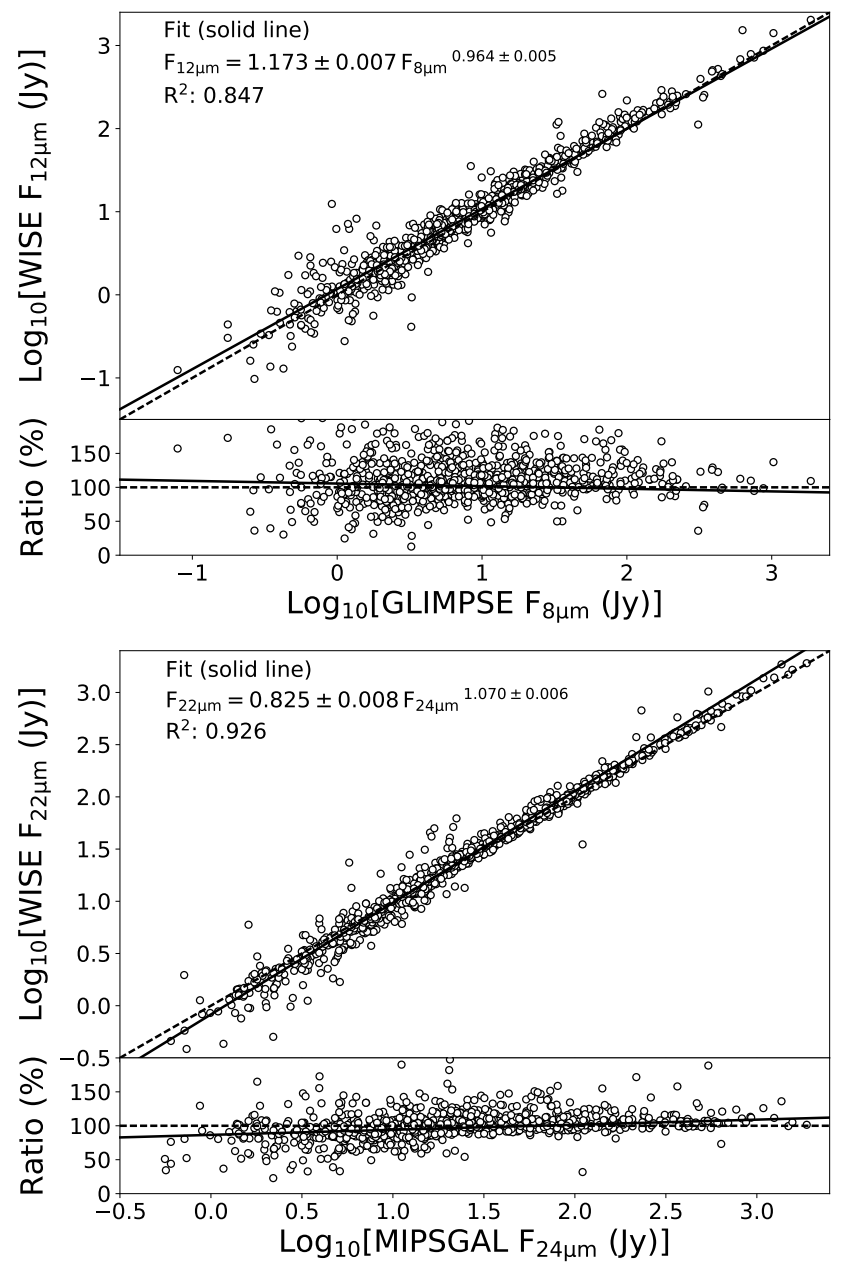

Figure 4. Correlations between similar IR photometric bands. The upper subplot shows the GLIMPSE and W3 flux densities, while the lower subplot shows the correlation between MIPSGAL and W4 flux densities. The black solid lines represent the best fit. As in Figure 3, the lower panels represent the variations of the data points (in \%) from the unity line ratio (black dashed lines).

larger than $6^{\prime}$. There are also $16 \mathrm{H}$ II regions in our sample with radii $>6^{\prime}$. The slightly different fit to large H II regions (dotted line) does not influence our results: the lack of an offset remains for both smaller and larger $\mathrm{H}$ II regions.

Decreasing the flux densities by the factor of $0.07 \mathrm{Jy} \operatorname{arcmin}^{-2}$ (middle subplot on Figure 5) results in a much poorer correlation. The standard deviation from the $1: 1$ line is $\sim 7$ times higher $(\sim 27.4 \mathrm{Jy})$ after applying the correction factor of $0.07 \mathrm{Jy} \operatorname{arcmin}^{-2}$ to our MAGPIS data points (bottom panel of the middle subplot on Figure 5$)$, than before ( $\sim 4.3 \mathrm{Jy}$; bottom panel of the top subplot on Figure 5). Worse, the flux densities for $42 \%$ of the regions become negative if the factor is applied, and only 5 of the 16 large regions has a positive flux density value.

Some scatter in the relationships may come from ra- 

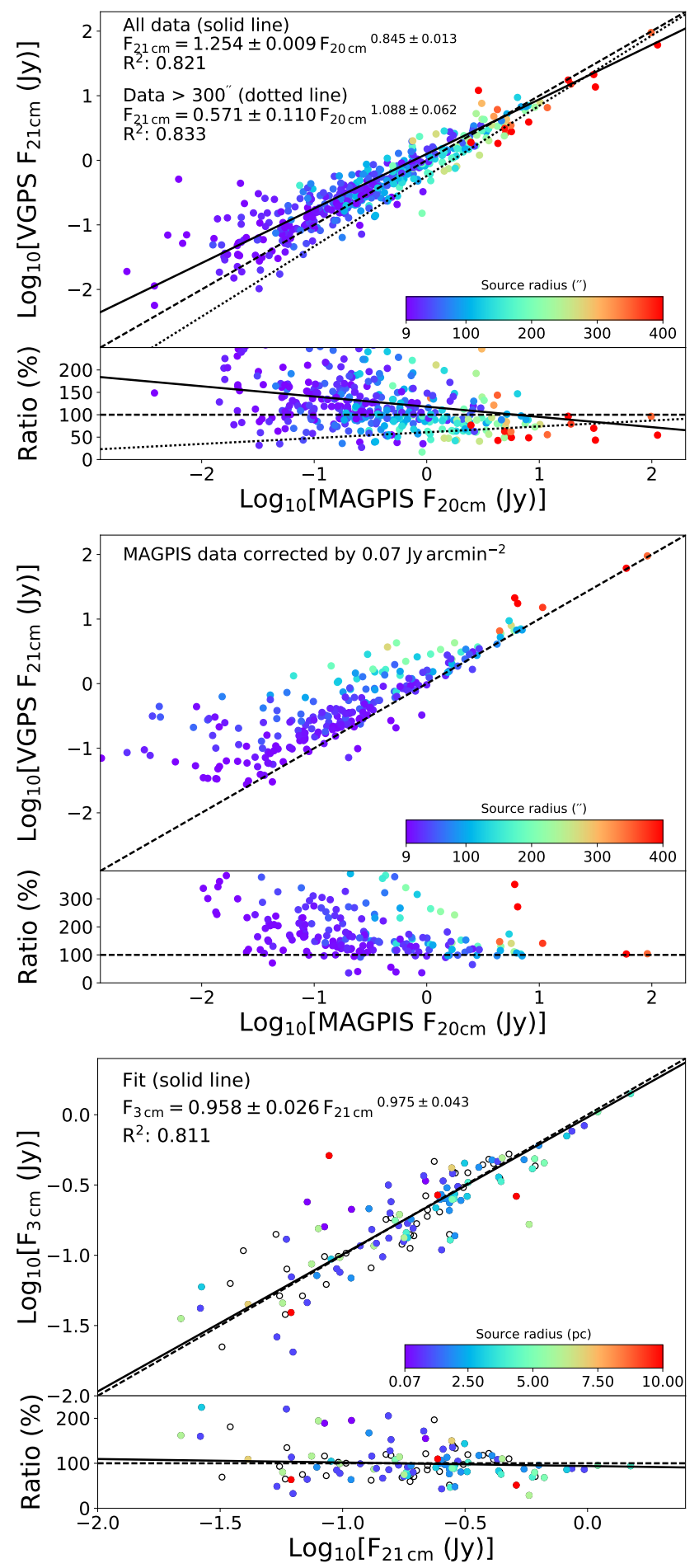

Figure 5. The correlations between VGPS and MAGPIS, and the HRDS and VGPS flux densities. The black dashed lines represent the 1:1 ratio. Top: shows a fit to all data points (solid line) and a fit to data from regions with radii $>6^{\prime}$ (dotted lines). Middle: comparison with the MAGPIS flux densities corrected by a factor of $0.07 \mathrm{Jy} \mathrm{arcmin}^{-2}$ (see text). Bottom: The open circles represent sources without distance measurements (no physical radius). The solid black line shows the best fit to the highest quality data (colored and empty circles). The correlation suggests negligible radio optical depth effects. dio optical depth effects. At low radio frequencies, the radio continuum emission from $\mathrm{H}$ II regions can be optically thick. The wavelength of this optically thick transition is determined by the electron density, and therefore young compact HII regions are more likely to be optically thick at a given frequency. Comparing $3 \mathrm{~cm}$ (HRDS) and $21 \mathrm{~cm}$ (VGPS) flux densities, it seems that the optical depth of regions does not strongly affect their flux densities (bottom subplot on Figure 5). We only compare flux densities for $\mathrm{H}$ II regions with HRDSderived radii that are within $50 \%$ of the values derived here, which ensures that we are sampling the same radio continuum sources. From Figure 5, it is clear that the strength of the correlation is the same, regardless of $\mathrm{H}$ II region physical radius. Because the exponent of the fit to the data is close to unity $(0.975 \pm 0.043)$, we conclude that statistically the optical depth of $\mathrm{H}$ II regions at $21 \mathrm{~cm}$ does not have a strong effect on the measured flux densities.

\subsection{Combined radio data set}

For much of the following analysis, we use a combined MAGPIS and VGPS data set. The two flux densities are strongly correlated (see Figure 5), and are at approximately the same wavelength. Some large diffuse sources are detected in the VGPS that are not in MAGPIS and many compact sources are unconfused in MAGPIS, but are confused in the VGPS. The combination allows us to determine radio flux densities for the largest possible sample. The combined data set has VGPS flux densities if those of MAGPIS are not available, and MAGPIS flux densities if those of the VGPS are not available. For sources that have both VGPS and MAGPIS flux densities, we use the average. We denote this combined data set using "radio", i.e. the combined flux density is given by the term $F_{\text {radio }}$.

\subsection{IR and radio flux density correlations}

We investigate correlations between the IR and radio flux densities in Figure 6, and summarize the results in Table 2, Figures 7 and 8.

The infrared and radio flux densities are strongly correlated, albeit with higher scatter at the lower flux density values. We perform each fit twice, once to all data (solid line in Figure 6), and once to data from regions with radii $r>1 \mathrm{pc}$ (dotted line in Figure 6). The latter fit by default excludes all regions lacking known distances. The fits to all data all have similar power law exponents in the range $\sim 0.7-0.8$. The power law exponents for regions with radii $r>1 \mathrm{pc}$ are all near unity. These results show that the smallest regions have on average higher IR to radio flux density ratios compared with larger regions. Additionally, there are many regions with low radio flux densities and high IR to radio 

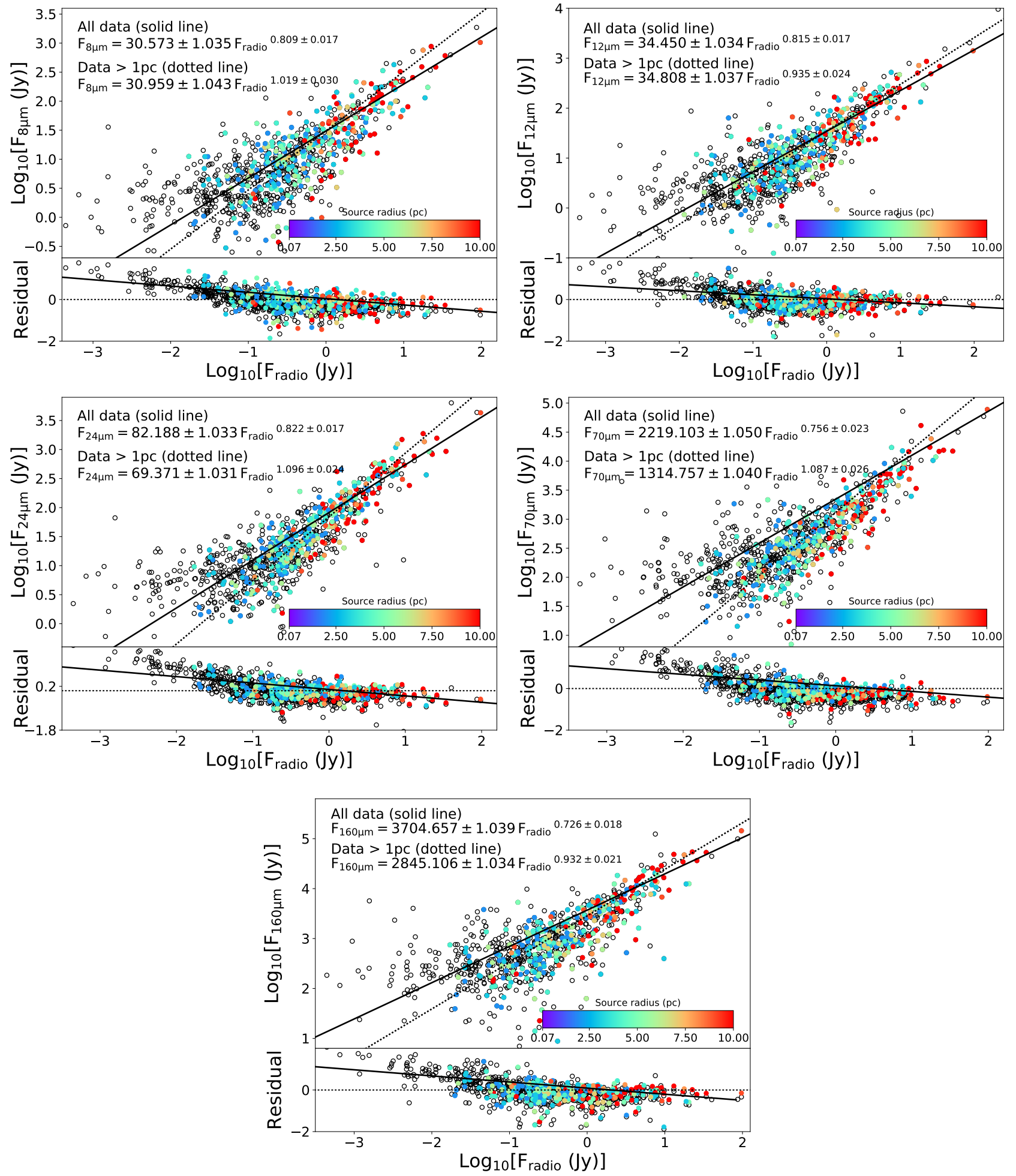

Figure 6. Comparisons of IR and radio flux densities for H II regions. The symbol colors reflect the physical radius in parsecs. The open circles are for sources that have no distance measurements, and hence unknown physical radii. The solid lines are the best fit to all data points. The dotted line marks the fit to the colored data points larger than 1 pc. The larger scatter at the lower flux densities probably caused by the photometric uncertainties. The label $F_{\text {radio }}$ denotes the combined $20 \mathrm{~cm}$ and $21 \mathrm{~cm}$ radio continumm data (see text). 
Table 2. Correlation between HII region radio and IR flux densities $^{\mathrm{a}}$

\begin{tabular}{ccrcc}
\hline \hline$\lambda(I R)$ & Data & \multicolumn{1}{c}{$A$} & $\alpha$ & $R^{2}$ \\
\hline \multirow{2}{*}{$8 \mu \mathrm{m}$} & All & $30.573 \pm 1.035$ & $0.809 \pm 0.017$ & 0.654 \\
& $>1 \mathrm{pc}$ & $30.959 \pm 1.043$ & $1.019 \pm 0.030$ & 0.385 \\
\multirow{2}{*}{$12 \mu \mathrm{m}$} & All & $34.450 \pm 1.034$ & $0.815 \pm 0.017$ & 0.670 \\
& $>1 \mathrm{pc}$ & $34.808 \pm 1.037$ & $0.935 \pm 0.024$ & 0.721 \\
\multirow{2}{*}{$24 \mathrm{~m}$} & All & $82.188 \pm 1.033$ & $0.822 \pm 0.017$ & 0.716 \\
& $>1 \mathrm{pc}$ & $69.371 \pm 1.031$ & $1.096 \pm 0.024$ & 0.526 \\
$70 \mu \mathrm{m}$ & All & $2219.103 \pm 1.050$ & $0.756 \pm 0.023$ & 0.421 \\
& $>1 \mathrm{pc}$ & $1314.757 \pm 1.040$ & $1.087 \pm 0.026$ & 0.369 \\
\multirow{2}{*}{$160 \mu \mathrm{m}$} & All & $3704.657 \pm 1.039$ & $0.726 \pm 0.018$ & 0.718 \\
& $>1 \mathrm{pc}$ & $2845.106 \pm 1.034$ & $0.932 \pm 0.021$ & 0.598 \\
\hline
\end{tabular}

${ }^{a}$ Fits made using equation $F_{\text {radio }}=A F_{\lambda(I R)}^{\alpha}$, where $F_{\text {radio }}$ denotes the combined $20 \mathrm{~cm}$ and $21 \mathrm{~cm}$ radio continuum data.

flux density ratios that lack physical size measurements; we speculate that these too have small physical sizes.

We observed similarly strong correlations between the IR and radio data with an exception of the $70 \mu \mathrm{m}$ data that has slightly weaker correlation. These strong correlations suggest that IR emissions can be used as a starformation tracer, observed even at longer wavelengths (e.g. Calzetti et al. 2010). When considering only the regions with radii $r>1 \mathrm{pc}$, the GLIMPSE $12.0 \mu \mathrm{m}$ and HiGal $160 \mu \mathrm{m}$ data show the strongest relationships. Since the radio emission is the most direct tracer of high-mass star-formation, these results hint that infrared emission can be used as a suitable proxy, but that the accuracy of the IR to radio ratio can be improved by excluding the smallest regions.

\subsection{Variation of IR and radio properties with HII region radius}

The size of an $\mathrm{H}$ II region is related to the ionizing photon flux (Strömgren 1939). H II regions expand as they age, but $\mathrm{H}$ II regions hosting more massive ionizing stars expand faster (cf. Spitzer 1978). On average, therefore, a population of larger H II regions would host stars with higher ionizing photon fluxes densities compared with a population of smaller H II regions.

We study the distribution of the IR to radio flux density ratios in Figure 7, and the IR flux density ratios (color indices) in Figure 8, all as a fuction of $\mathrm{H}$ II region radius. We summarize the results of these two Figures in Table 3, and graphically in Figure 9. In this analysis, for clarity we do not analyze the $22 \mu \mathrm{m}$ data since the flux densities are essentially the same as those at $24 \mu \mathrm{m}$ (see Figure 4). Table 3 gives the median values of the flux densities (dashed lines on Figures 7), the standard deviations, the color criteria, that is, the range of the "whiskers" (see subtitle of Figure 7) in Figures 7 and 8, and the number of data points in four different source size bins. The lines "All" show the same values but for all data points, independent of the bins.

The IR to radio flux density ratios in Figure 7 and Table 3 show that the median IR to radio ratio has no strong dependence on H II region size. We note, however, that the median IR to radio ratios for $24 \mu \mathrm{m}, 70 \mu \mathrm{m}$, and $160 \mu \mathrm{m}$ is elevated for the smallest regions with $r<1 \mathrm{pc}$. Additionally, the spread of IR to radio ratios is larger for all IR wavelengths in this smallest size bin.

We investigate the IR colors of $\mathrm{H}$ II regions as a function of $\mathrm{H}$ II region radius in Figure 8, summarize the results in Figure 9, and list the results in Table 3. In this table, we give the median values of the flux densities (red lines on Figures 7 and 8), the standard deviations, the color criteria (range between the whiskers) and the number of data points in four different source size bins. The lines "All" show the same values but for all data points, independent of the bins. Despite the strong correlation of $\mathrm{H}$ II region flux densities and IR to radio ratios with $\mathrm{H}$ II region sizes, IR colors are not a strong function of $\mathrm{H}$ II region size. The two exceptions to this trend are the $\log _{10}\left(F_{70 \mu \mathrm{m}} / F_{24 \mu \mathrm{m}}\right)$ ratio, which does decrease with increasing H II region size, and the $\log _{10}\left(F_{160 \mu \mathrm{m}} / F_{70 \mu \mathrm{m}}\right)$ ratio, which increases with increasing $\mathrm{H}$ II region size. Because the $70 \mu \mathrm{m}$ data are sensitive to both small and large grains, the dependence of this ratio on $\mathrm{H}$ II region size may imply that the larger regions have a larger small grain population relative to large grains. It is also worth noting that the $\log _{10}\left(F_{160 \mu \mathrm{m}} / F_{70 \mu \mathrm{m}}\right)$ ratio is slightly above unity for all sizes, which implies that the peak of the spectral energy distribution is closer to $160 \mu \mathrm{m}$ than $70 \mu \mathrm{m}$ (Anderson et al. 2012).

Dopita et al. (2006) suggested that infrared color-color diagrams of $\mathrm{H}$ II regions can be used as a pressure diagnostic tool. They produced color-color diagrams (Figure 11 in their paper) using theoretical spectral energy distributions (SEDs) passed through the transmission function of the MIPS instrument on Spitzer. We were not able to compare the theoretical models introduced by Dopita et al. (2006) with our observational data points because their SEDs peak at $\sim 30 \mu \mathrm{m}$; a peak this low is not observed.

\subsection{Do the UC HII region color criteria only select UC HII regions?}

It has been known for some time that IR colors can be used to identify H II regions (Wood \& Churchwell 1989b; Hughes \& MacLeod 1989; Zoonematkermani et al. 1990; White et al. 1991; Helfand et al. 1992; Becker et al. 1994). The IR criteria, however, have been derived almost exclusively using IRAS point sources. At the IRAS angular resolutions of $0.5,0.5,1.0$ and 2.0 at $12 \mu \mathrm{m}$, 

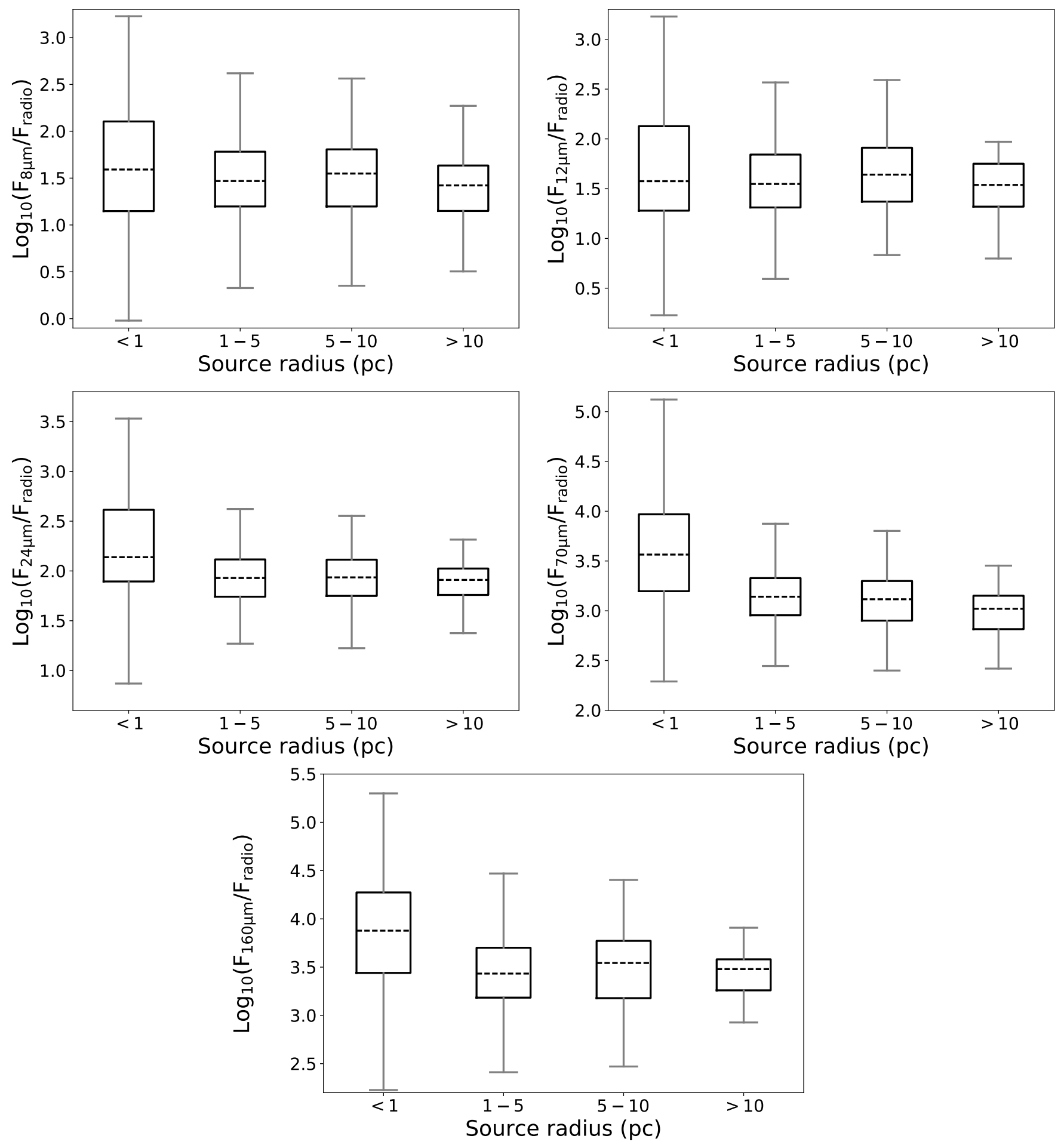

Figure 7. "Box and whiskers" plots for the IR/radio ratios in four size bins. The dashed lines mark the median values (second quartile). The lower and upper limits of the boxes mark the $25^{t h}$ percentile (first quartile; Q1) and $75^{t h}$ percentile (third quartile; Q3) of the data, respectively. We use for the "whiskers": $Q 1-1.5 \times I Q R$ (for the lower limit) and $Q 3+1.5 \times I Q R$ (for the upper limit), where IQR is the interquartile $(I Q R=Q 3-Q 1)$. The plots show relatively flat IR to radio ratios as a function of $\mathrm{H}$ II region size, although the ratio is elevated for the smallest H II regions $<1 \mathrm{pc}$ at the longer IR wavelengths. The label $F_{\text {radio }}$ denotes the combination of the $20 \mathrm{~cm}$ and the $21 \mathrm{~cm}$ radio continuum data (see Section 4.3 ). 

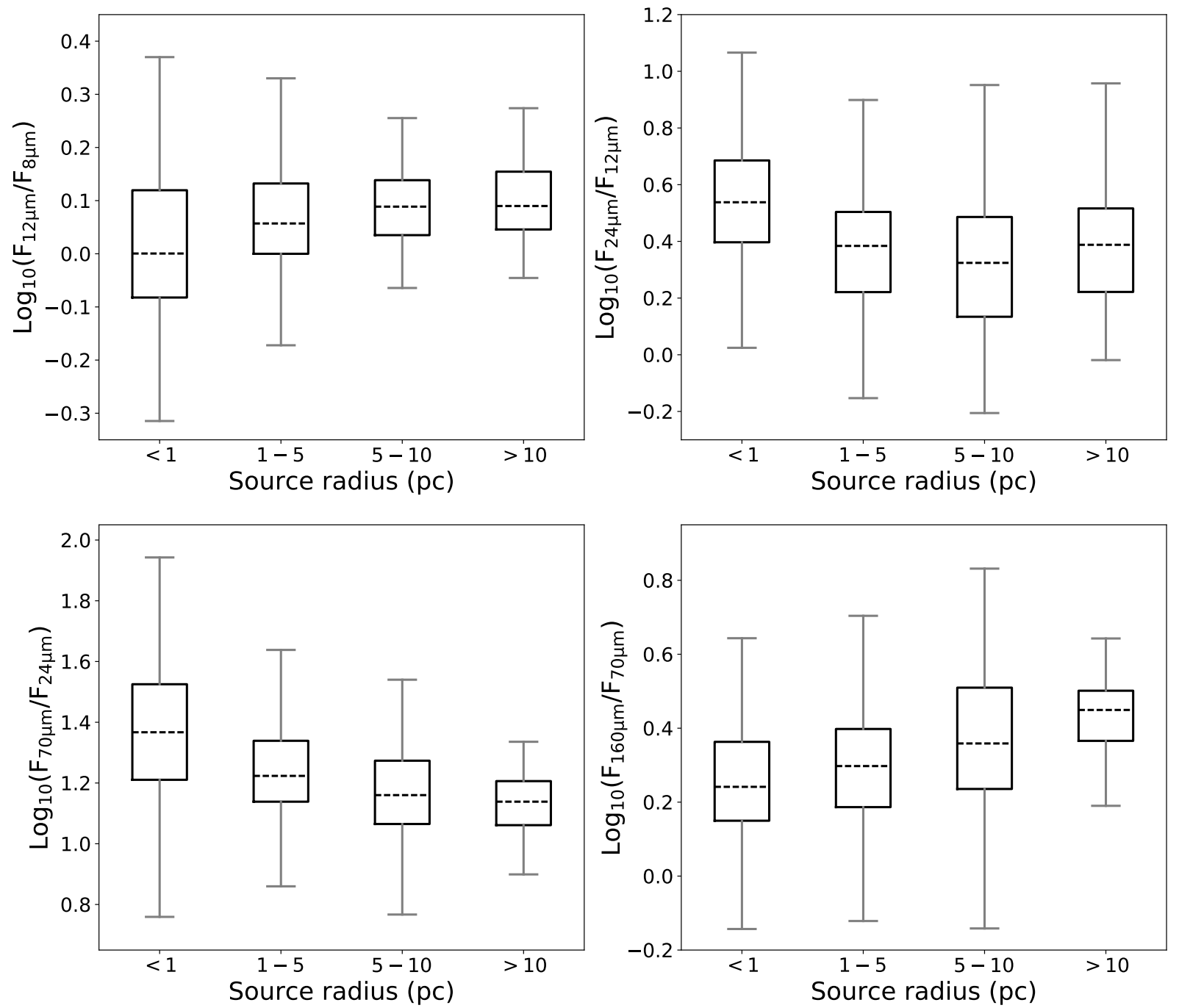

Figure 8. Box and whiskers plots as in Figure 7 for the IR colors in four size bins. The $12 \mu \mathrm{m}$ to $8 \mu \mathrm{m}$ and $24 \mu \mathrm{m}$ to $12 \mu \mathrm{m}$ ratios do not show a clear trend. The $70 \mu \mathrm{m}$ to $24 \mu \mathrm{m}$ ratio decreasing with increasing H II region radius, while the $160 \mu \mathrm{m}$ to $70 \mu \mathrm{m}$ ratio increases with $\mathrm{H}$ II region radius. 
Table 3. Median IR and radio flux density ratios as a function of H II region radius

\begin{tabular}{|c|c|c|c|c|c|}
\hline Flux density ratio & $\begin{array}{l}\text { Radius } \\
\text { [pc] }\end{array}$ & Median & $\sigma$ & $\begin{array}{c}\text { Color criteria } \\
(\mathrm{Q} 1-1.5 \times \mathrm{IQR}-\mathrm{Q} 3+1.5 \times \mathrm{IQR})^{*}\end{array}$ & \# of sources \\
\hline \multirow{5}{*}{$\log _{10}\left(\mathrm{~F}_{8 \mu \mathrm{m}} / \mathrm{F}_{\text {radio }}\right)$} & $r<1$ & 1.59 & 0.42 & $-0.02-3.23$ & 141 \\
\hline & $1<r<5$ & 1.47 & 0.31 & $0.33-2.62$ & 237 \\
\hline & $5<r<10$ & 1.55 & 0.35 & $0.35-2.56$ & 87 \\
\hline & $r>10$ & 1.42 & 0.27 & $0.50-2.27$ & 43 \\
\hline & All & 1.52 & 0.34 & $-0.02-3.23$ & 508 \\
\hline \multirow{5}{*}{$\log _{10}\left(\mathrm{~F}_{12 \mu \mathrm{m}} / \mathrm{F}_{\text {radio }}\right)$} & $r<1$ & 1.57 & 0.56 & $0.23-3.23$ & 137 \\
\hline & $1<r<5$ & 1.55 & 0.29 & $0.59-2.57$ & 238 \\
\hline & $5<r<10$ & 1.67 & 0.24 & $0.83-2.59$ & 98 \\
\hline & $r>10$ & 1.54 & 0.19 & $0.80-1.97$ & 48 \\
\hline & All & 1.59 & 0.32 & $0.23-3.23$ & 521 \\
\hline \multirow{5}{*}{$\log _{10}\left(\mathrm{~F}_{24 \mu \mathrm{m}} / \mathrm{F}_{\text {radio }}\right)$} & $r<1$ & 2.14 & 0.46 & $0.87-3.53$ & 136 \\
\hline & $1<r<5$ & 1.93 & 0.19 & $1.27-2.62$ & 232 \\
\hline & $5<r<10$ & 1.94 & 0.17 & $1.22-2.55$ & 90 \\
\hline & $r>10$ & 1.91 & 0.11 & $1.37-2.31$ & 42 \\
\hline & All & 1.99 & 0.25 & $0.87-3.53$ & 500 \\
\hline \multirow{5}{*}{$\log _{10}\left(\mathrm{~F}_{70 \mu \mathrm{m}} / \mathrm{F}_{\text {radio }}\right)$} & $r<1$ & 3.56 & 0.36 & $2.29-5.12$ & 142 \\
\hline & $1<r<5$ & 3.14 & 0.19 & $2.45-3.87$ & 240 \\
\hline & $5<r<10$ & 3.12 & 0.22 & $2.40-3.80$ & 95 \\
\hline & $r>10$ & 3.02 & 0.20 & $2.42-3.45$ & 44 \\
\hline & All & 3.20 & 0.24 & $2.29-5.12$ & 521 \\
\hline \multirow{5}{*}{$\log _{10}\left(\mathrm{~F}_{160 \mu \mathrm{m}} / \mathrm{F}_{\text {radio }}\right)$} & $r<1$ & 3.88 & 0.44 & $2.23-5.30$ & 140 \\
\hline & $1<r<5$ & 3.43 & 0.25 & $2.41-4.47$ & 239 \\
\hline & $5<r<10$ & 3.54 & 0.36 & $2.47-4.40$ & 91 \\
\hline & $r>10$ & 3.48 & 0.18 & $2.93-3.91$ & 42 \\
\hline & All & 3.59 & 0.31 & $2.23-5.30$ & 512 \\
\hline \multirow{5}{*}{$\log _{10}\left(\mathrm{~F}_{12 \mu \mathrm{m}} / \mathrm{F}_{8 \mu \mathrm{m}}\right)$} & $r<1$ & 0.00 & 0.12 & $-0.31-0.37$ & 327 \\
\hline & $1<r<5$ & 0.06 & 0.07 & $0.17-0.33$ & 267 \\
\hline & $5<r<10$ & 0.09 & 0.05 & $-0.06-0.26$ & 91 \\
\hline & $r>10$ & 0.09 & 0.06 & $-0.05-0.27$ & 46 \\
\hline & All & 0.06 & 0.07 & $-0.31-0.37$ & 550 \\
\hline \multirow{5}{*}{$\log _{10}\left(\mathrm{~F}_{24 \mu \mathrm{m}} / \mathrm{F}_{12 \mu \mathrm{m}}\right)$} & $r<1$ & 0.54 & 0.14 & $0.02-1.07$ & 144 \\
\hline & $1<r<5$ & 0.38 & 0.16 & $-0.15-0.90$ & 262 \\
\hline & $5<r<10$ & 0.32 & 0.19 & $-0.21-0.95$ & 92 \\
\hline & $r>10$ & 0.39 & 0.17 & $-0.02-0.96$ & 44 \\
\hline & All & 0.41 & 0.16 & $-0.21-1.07$ & 542 \\
\hline
\end{tabular}


Table 3 (continued)

\begin{tabular}{cccccc}
\hline \hline Flux density ratio & $\begin{array}{c}\text { Radius } \\
{[\mathrm{pc}]}\end{array}$ & Median & $\sigma$ & $\begin{array}{c}\text { Color criteria } \\
(\mathrm{Q} 1-1.5 \times \mathrm{IQR}-\mathrm{Q} 3+1.5 \times \mathrm{IQR})^{*}\end{array}$ & \# of sources \\
\hline & & & & & \\
& $r<1$ & 1.37 & 0.16 & $0.76-1.94$ & 145 \\
& $1<r<5$ & 1.22 & 0.12 & $0.86-1.64$ & 262 \\
$\log _{10}\left(\mathrm{~F}_{70 \mu \mathrm{m}} / \mathrm{F}_{24 \mu \mathrm{m}}\right)$ & $5<r<10$ & 1.16 & 0.38 & $0.77-1.54$ & 45 \\
& $r>10$ & 1.14 & 0.20 & $0.90-1.34$ & 545 \\
& All & 1.23 & 0.21 & $0.76-1.94$ & 150 \\
& & & & & 273 \\
$\log _{10}\left(\mathrm{~F}_{160 \mu \mathrm{m}} / \mathrm{F}_{70 \mu \mathrm{m}}\right)$ & $5<r<1$ & 0.24 & 0.12 & $-0.14-0.64$ & 46 \\
& $\quad r<10$ & 0.36 & 0.15 & $-0.12-0.70$ & 563 \\
\hline
\end{tabular}

* $Q 1$ and $Q 3$ are the $25^{t} h$ and $75^{t} h$ percentile of the data, respectively, and $I Q R=(Q 3-Q 1)$ is the interquartile.

$25 \mu \mathrm{m}, 60 \mu \mathrm{m}$ and $100 \mu \mathrm{m}$ respectively, the use of these IRAS criteria only identify relatively compact sources. WC89 suggested that the IR colors of ultra-compact (UC) H II regions are distinct from other sources found in the IRAS PSC, which was supported by high resolution VLA observations (Wood \& Churchwell 1989b). They found that UC HII regions have color indices of $\log _{10}\left(F_{25 \mu \mathrm{m}} / F_{12 \mu \mathrm{m}}\right) \geq 0.57$ and $\log _{10}\left(F_{60 \mu \mathrm{m}} / F_{12 \mu \mathrm{m}}\right) \geq$ 1.22. Based on the number of IRAS PSC sources that satisfy these criteria, they suggested that there are 1650 UC HII regions in the Galaxy. By examining the IRAS colors of optical H II regions, Hughes \& MacLeod (1989) found that similar colors select UC H II regions: $\log _{10}\left(F_{25 \mu \mathrm{m}} / F_{12 \mu \mathrm{m}}\right) \geq 0.4$ and $\log _{10}\left(F_{60 \mu \mathrm{m}} / F_{25 \mu \mathrm{m}}\right) \geq$ 0.25 and estimated that there are $\sim 2300 \mathrm{UC} \mathrm{H}$ II regions in the Galaxy.

It is often assumed that the WC89 criteria identify UC H II regions, but perhaps not more evolved nebulae (e.g. Cohen et al. 2007). Although there is evidence that all $\mathrm{H}$ II regions have similar IRAS colors (Hughes \& MacLeod 1989; Anderson et al. 2012), this has never been proven for a sample as large as ours. Chini et al. (1987) found in a study of 66 compact H II regions that all had a similar energy distributions in the range $1-$ $1300 \mu \mathrm{m}$. Leto et al. (2009) found that UC H II regions cannot be identified solely by their infrared colors due to the contamination of other classes of Galactic sources. The number of UC H II regions in the Galaxy therefore remains somewhat uncertain.

In Figure 10 we show similar color-color diagrams to those of WC89 (their Figure 1b): in place of their $\log _{10}\left(F_{25 \mu \mathrm{m}} / F_{12 \mu \mathrm{m}}\right)$ and $\log _{10}\left(F_{60 \mu \mathrm{m}} / F_{12 \mu \mathrm{m}}\right)$ color indices we show $\log _{10}\left(F_{24 \mu \mathrm{m}} / F_{12 \mu \mathrm{m}}\right)$ and $\log _{10}\left(F_{70 \mu \mathrm{m}} / F_{12 \mu \mathrm{m}}\right)$ color indices. Since our $70 \mu \mathrm{m}$ data sample a slightly different portion of the spectral energy distribution compared with IRAS $60 \mu \mathrm{m}$ data, we must scale the WC89 criteria used to identify UC H II regions. The PACS guide ${ }^{7}$ shows that the PACS $70 \mu \mathrm{m}$ data should be multipled by 0.76 to convert to the IRAS $60 \mu \mathrm{m}$ bandpass, assuming 30 to $100 \mathrm{~K}$ dust. The WC89 criterion of $\log _{10}\left(F_{60 \mu \mathrm{m}} / F_{12 \mu \mathrm{m}}\right) \geq 1.22$ therefore is equivalent to $\log _{10}\left(F_{70 \mu \mathrm{m}} / F_{12 \mu \mathrm{m}}\right) \geq 1.34$, which we show on the plot. Because of the similarity of the bandpasses, we do not scale the $12 \mu \mathrm{m}$ or $25 \mu \mathrm{m}$ flux densities. As we do not have flux densities at $100 \mu \mathrm{m}$, we are not able to reproduce the $\log _{10}\left(F_{25 \mu \mathrm{m}} / F_{12 \mu \mathrm{m}}\right)$ versus $\log _{10}\left(F_{100 \mu \mathrm{m}} / F_{60 \mu \mathrm{m}}\right)$ color-color plot from WC89. Instead, we show $\log _{10}\left(F_{24 \mu \mathrm{m}} / F_{12 \mu \mathrm{m}}\right)$ versus $\log _{10}\left(F_{160 \mu \mathrm{m}} / F_{70 \mu \mathrm{m}}\right)$.

Figure 10 shows that the color citeria established by WC89 are most sensitive to the smallest regions, but do not uniquely identify UC H II regions. In the size bins defined earlier, $\sim 43 \%, \sim 12 \%, \sim 16 \%$ and $\sim 11 \%$ of colored data points satisfy the $\log _{10}\left(F_{24 \mu \mathrm{m}} / F_{12 \mu \mathrm{m}}\right)$ versus $\log _{10}\left(F_{70 \mu \mathrm{m}} / F_{12 \mu \mathrm{m}}\right)$ color criteria of WC89 for source radius bins of $r<1 \mathrm{pc}, 1<r<5 \mathrm{pc}, 5<r<10 \mathrm{pc}$

\footnotetext{
7 http://herschel.esac.esa.int/twiki/pub/Public/ PacsCalibrationWeb/cc_report_v1.pdf
} 

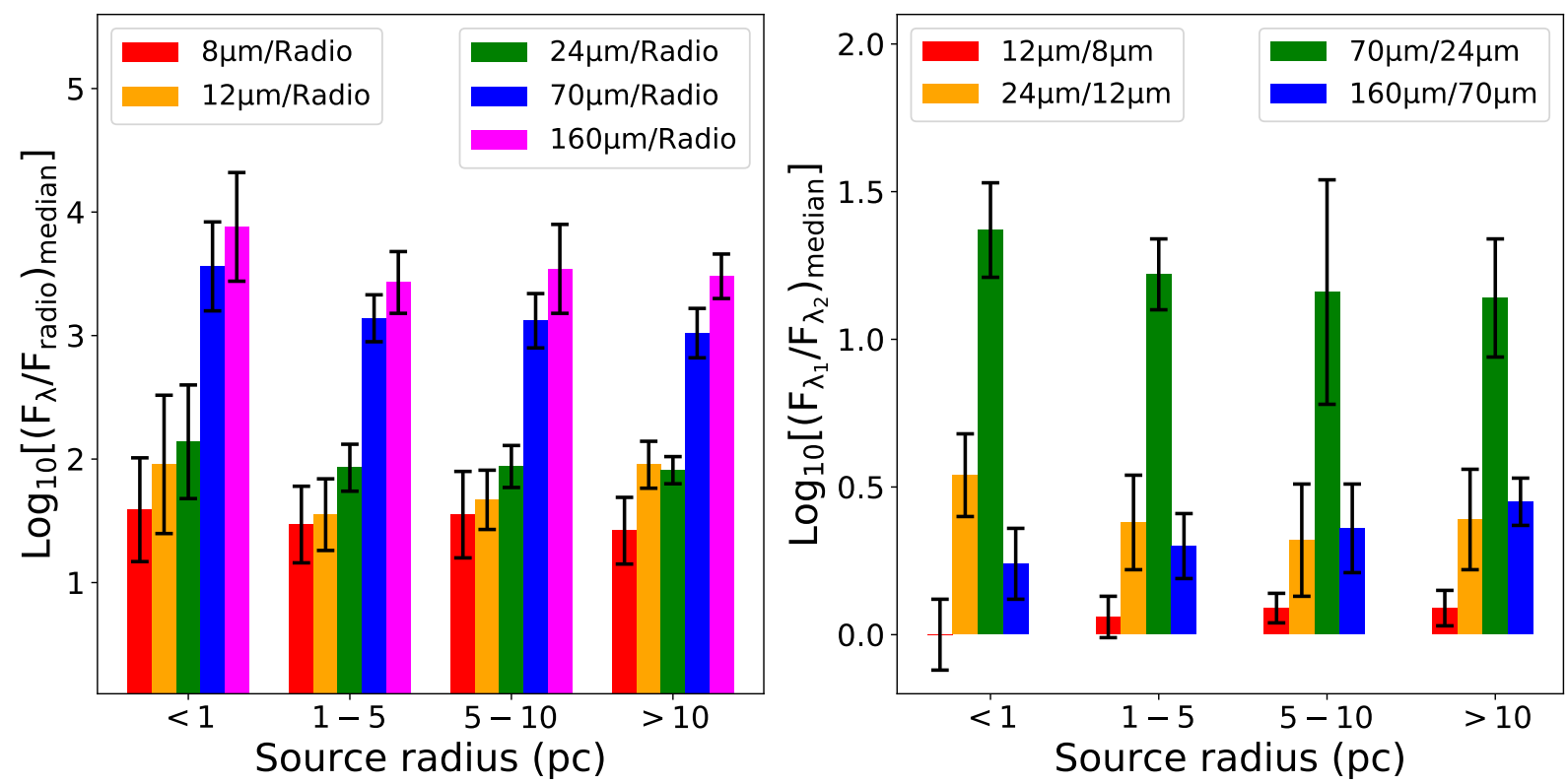

Figure 9. Graphical summary of the median values from Table 3. The standard deviation of the data points is marked with the error bars. The "radio" term means the combination of the $20 \mathrm{~cm}$ and the $21 \mathrm{~cm}$ radio continuum data, see Section 4.3 . On the left plot, we show the ratios of the IR and radio flux densities. For the smallest regions, the IR to radio ratios are higher at the longest wavelengths, and the dispersion is greater. The right plot shows the distribution of median values of color indices. This plot suggests that all the investigated IR flux density ratios are unchanged within the errors, independent of H II region size.

and $r>10 \mathrm{pc}$, respectively. For the color indices $\log _{10}\left(F_{24 \mu \mathrm{m}} / F_{12 \mu \mathrm{m}}\right)$ versus $\log _{10}\left(F_{160 \mu \mathrm{m}} / F_{70 \mu \mathrm{m}}\right)$, due to the uncertainty of converting the IRAS color criteria to the wavelengths used here, we can not reliably give percentages. Although there are no definite H II region criteria for the $\log _{10}\left(F_{24 \mu \mathrm{m}} / F_{12 \mu \mathrm{m}}\right)$ versus $\log _{10}\left(F_{160 \mu \mathrm{m}} / F_{70 \mu \mathrm{m}}\right)$ color indices, the distribution of data points in our plot looks similar to that of WC89, and suggest no visible trend with $\mathrm{H}$ II region size.

The new IR color indices defined here (solid lines in Figure 10), $\log _{10}\left(F_{24 \mu \mathrm{m}} / F_{12 \mu \mathrm{m}}\right) \geq 0$ and $\log _{10}\left(F_{70 \mu \mathrm{m}} / F_{12 \mu \mathrm{m}}\right) \geq 1.2$, are satisfied by $\sim 96 \%$, $\sim 94 \%, \sim 95 \%$ and $\sim 98 \%$ of the colored data points for source radii bins of $r<1 \mathrm{pc}, 1<r<5 \mathrm{pc}, 5<r<10 \mathrm{pc}$ and $r>10 \mathrm{pc}$, respectively. For the IR color limits of $\log _{10}\left(F_{24 \mu \mathrm{m}} / F_{12 \mu \mathrm{m}}\right) \geq 0$ and $\log _{10}\left(F_{160 \mu \mathrm{m}} / F_{70 \mu \mathrm{m}}\right) \leq$ 0.67 , these numbers are $\sim 98 \%, \sim 94 \%, \sim 87 \%$ and $\sim 91 \%$ for the same source radii bins.

Dreher \& Welch (1981) suggested that the number of UC HII regions is much higher in our Galaxy then we would expect if we assume that their sizes are determined by a free expansion at the thermal sound speed, leading to the so-called "lifetime problem". The estimates for the number of UC H II regions in the Galaxy by WC89 and Hughes \& MacLeod (1989) only exacerbated the lifetime problem. There have been a number of solutions to the lifetime problem proposed. For example, Peters et al. (2010a,b, and references therein), showed that the HII regions do not expand monotonically and isotropically (during the main accretion phase). This effect causes flickering of the radio continuum emission, which has been observed in Sgr B2 by De Pree et al. (2014). It remains unclear, however, if the lifetime problem is as acute as suggested due to uncertainties in the number of UC HII regions in the Galaxy. The similarity of all $\mathrm{HII}$ region IR colors, regardless of radius, implies that perhaps the number of UC HII regions in the Galaxy has been overestimated, and therefore the lifetime problem is much less severe than sometimes assumed.

The radii we use here cannot be directly compared with those derived previously for UC HII regions. Churchwell (2002) state that UC H II regions have diameters $\lesssim 0.1 \mathrm{pc}$. These sizes, however, correspond to the densest parts of ionized gas, sampled with highresolution radio interferometric observations. Our sizes include all the emission from the regions, including any possible extended envelopes (Kim \& Koo 2002). Nevertheless, the fact that nearly $25 \%$ of the H II regions in our sample satisfy the color indices, and that our sample includes all known Galactic H II regions, means that the criteria cannot uniquely identify UC H II regions. The low total number of H II regions satisfying the WC89 criteria, and the fact that these criteria do not select only UC H II regions, implies a significant uncertainty in the number of UC H II regions in the Galaxy derived using the WC89 criteria.

Previous studies of IR colors of galaxies showed characteristic values for the IRAS bands. For example, Helou (1986) examined galaxies with no quasar- 

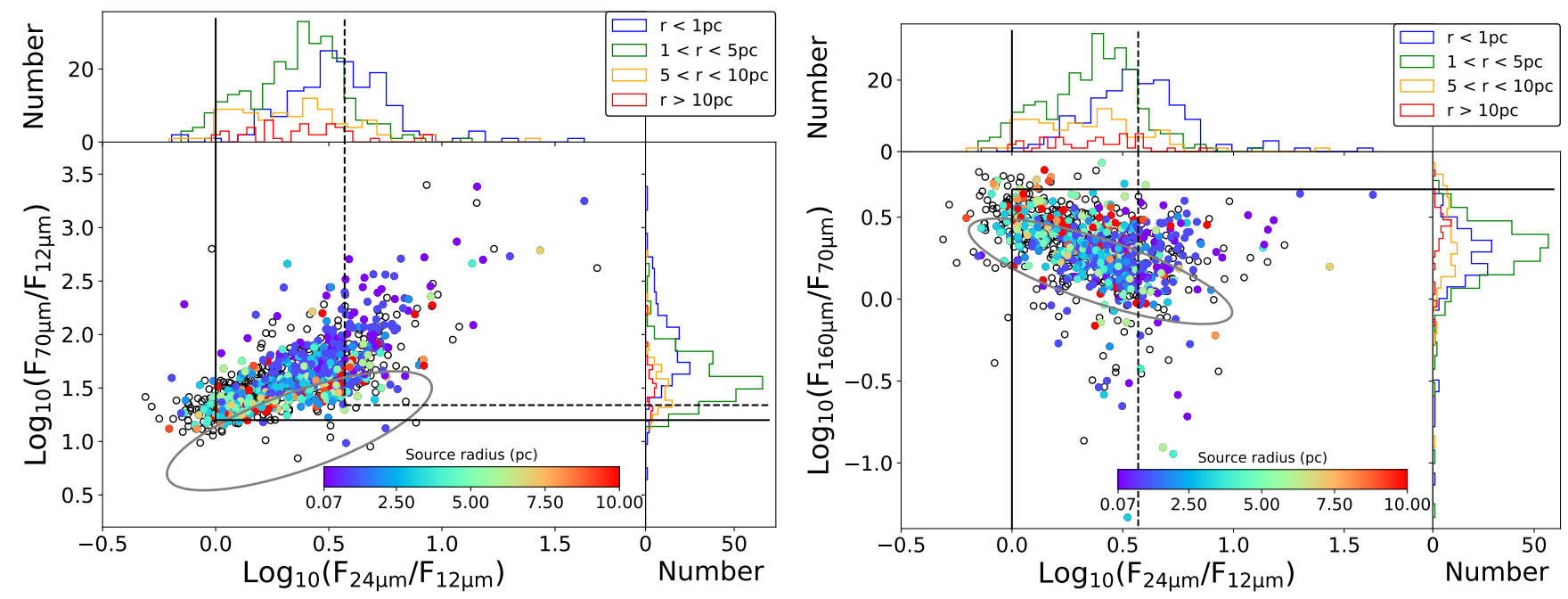

Figure 10. H II region color-color diagrams similar to those in WC89. The color scale shows the physical size of the H II regions in parsecs while the empty black circles represent the sources lacking size measurements. On the left subplot, the dashed lines represent the color-corrected infrared color indices $\log _{10}\left(F_{25 \mu \mathrm{m}} / F_{12 \mu \mathrm{m}}\right) \geq 0.57$ and $\log _{10}\left(F_{70} \mu \mathrm{m} / F_{12} \mu \mathrm{m}\right) \geq 1.34$ used to separate the UC H II regions from other point sources (WC89). On the right subplot, the dashed line shows the $\log _{10}\left(F_{25} \mu \mathrm{m} / F_{12} \mu \mathrm{m}\right) \geq 0.57$ limit. The gray ellipses show areas where $\sim 95 \%$ of the galaxies from Sanders et al. (2003) are located (see text). The solid lines represent the criteria suggested by our data: $\log _{10}\left(F_{24 \mu \mathrm{m}} / F_{12 \mu \mathrm{m}}\right) \geq 0$ and $\log _{10}\left(F_{70 \mu \mathrm{m}} / F_{12 \mu \mathrm{m}}\right) \geq 1.2$ (left subplot), $\log _{10}\left(F_{24 \mu \mathrm{m}} / F_{12 \mu \mathrm{m}}\right) \geq 0$ and $\log _{10}\left(F_{160 \mu \mathrm{m}} / F_{70 \mu \mathrm{m}}\right) \leq 0.67$ (right subplot). These color indices can therefore be used as an approximate IR limit for H II regions, independent of size.

like nucleus and found a clear decreasing trend in $\log _{10}\left(F_{60 \mu \mathrm{m}} / F_{100 \mu \mathrm{m}}\right)$ as the ratio $\log _{10}\left(F_{12 \mu \mathrm{m}} / F_{25 \mu \mathrm{m}}\right)$ increases. This indicates a higher star-formation rate at higher values of $\log _{10}\left(F_{60 \mu \mathrm{m}} / F_{100 \mu \mathrm{m}}\right)$ and lower values of $\log _{10}\left(F_{12 \mu \mathrm{m}} / F_{25 \mu \mathrm{m}}\right)$. Similar results have been reported by Soifer \& Neugebauer (1991); Wang (1991) and Sanders et al. (2003).

In Figure 10, we investigated the IR flux density ratio pairs of $\log _{10}\left(F_{24 \mu \mathrm{m}} / F_{12 \mu \mathrm{m}}\right)$ versus $\log _{10}\left(F_{160 \mu \mathrm{m}} / F_{70 \mu \mathrm{m}}\right), \quad$ and $\quad \log _{10}\left(F_{24 \mu \mathrm{m}} / F_{12 \mu \mathrm{m}}\right)$ versus $\log _{10}\left(F_{70 \mu \mathrm{m}} / F_{12 \mu \mathrm{m}}\right)$, using $\sim 600$ galaxies from Sanders et al. (2003). Their sample has galaxies of morphological types irregular and spiral, including starbursting. They found that the main contributor of the observed thermal emission from galaxies is the cool dust $\left(T_{\text {dust }} \sim 15-70 \mathrm{~K}\right)$. However, as Sanders et al. (2003) pointed out, a significant amount of emission from warm dust (peaking around $25 \mu \mathrm{m}$ ) has also been observed from galaxies. Sanders et al. (2003) also reported an inverse correlation between $\log _{10}\left(F_{12 \mu \mathrm{m}} / F_{25 \mu \mathrm{m}}\right)$ and $\log _{10}\left(F_{60 \mu \mathrm{m}} / F_{100 \mu \mathrm{m}}\right)$, which was interpreted as the result of efficient destruction of small grains due to the increasing total galaxy IR luminosity.

As we do not have the same IR wavelengths as IRAS, we require a correction factor to be able to compare with previous results. Using the PACS guide ${ }^{8}$, and assuming dust temperature range of $30-100 \mathrm{~K}$, we divided the flux density in the $60 \mu \mathrm{m}$ bandpass by a correction factor (0.76) to convert it to the $70 \mu \mathrm{m}$ bandpass. To convert the IRAS $100 \mu \mathrm{m}$ flux densities to the $160 \mu \mathrm{m}$ bandpass, we used SED templates of different type of galaxies from (Siebenmorgen \& Krügel 2007): $F_{160 \mu \mathrm{m}} \approx F_{100 \mu \mathrm{m}} / c f$ where $c f \approx 1.55$ is the averaged correction factor derived from the SEDs. Even if this method may not be sophisticated for qualitative studies, we believe, it is good enough for statistical purposes. About 95\% of the galaxies from Sanders et al. (2003) are located within the gray ellipses in Figure 10.

We conclude that the color indices of star forming galaxies are similar to those of $\mathrm{HII}$ regions. The $\log _{10}\left(F_{24} \mu \mathrm{m}\right) / \log _{10}\left(F_{12 \mu \mathrm{m}}\right)$ color index of H II regions is essentially the same as that of galaxies. There are offsets compare to the IR colors of Galactic H II regions ( 0.6 dex and $\sim 0.2$ dex in ratios of $\log _{10}\left(F_{70 \mu \mathrm{m}} / F_{12 \mu \mathrm{m}}\right)$ and $\log _{10}\left(F_{24 \mu \mathrm{m}} / F_{12 \mu \mathrm{m}}\right)$, respectively). Since the flux density of $\mathrm{HII}$ regions peaks near $70 \mu \mathrm{m}$, that this ratio differs between galaxies and HII regions is not unexpected.

\subsection{Variation of $I R$ and radio properties with $R_{\text {gal }}$}

We analyze the IR to radio flux density ratios (Figure 11), and the IR flux density ratios (Figure 12), as 

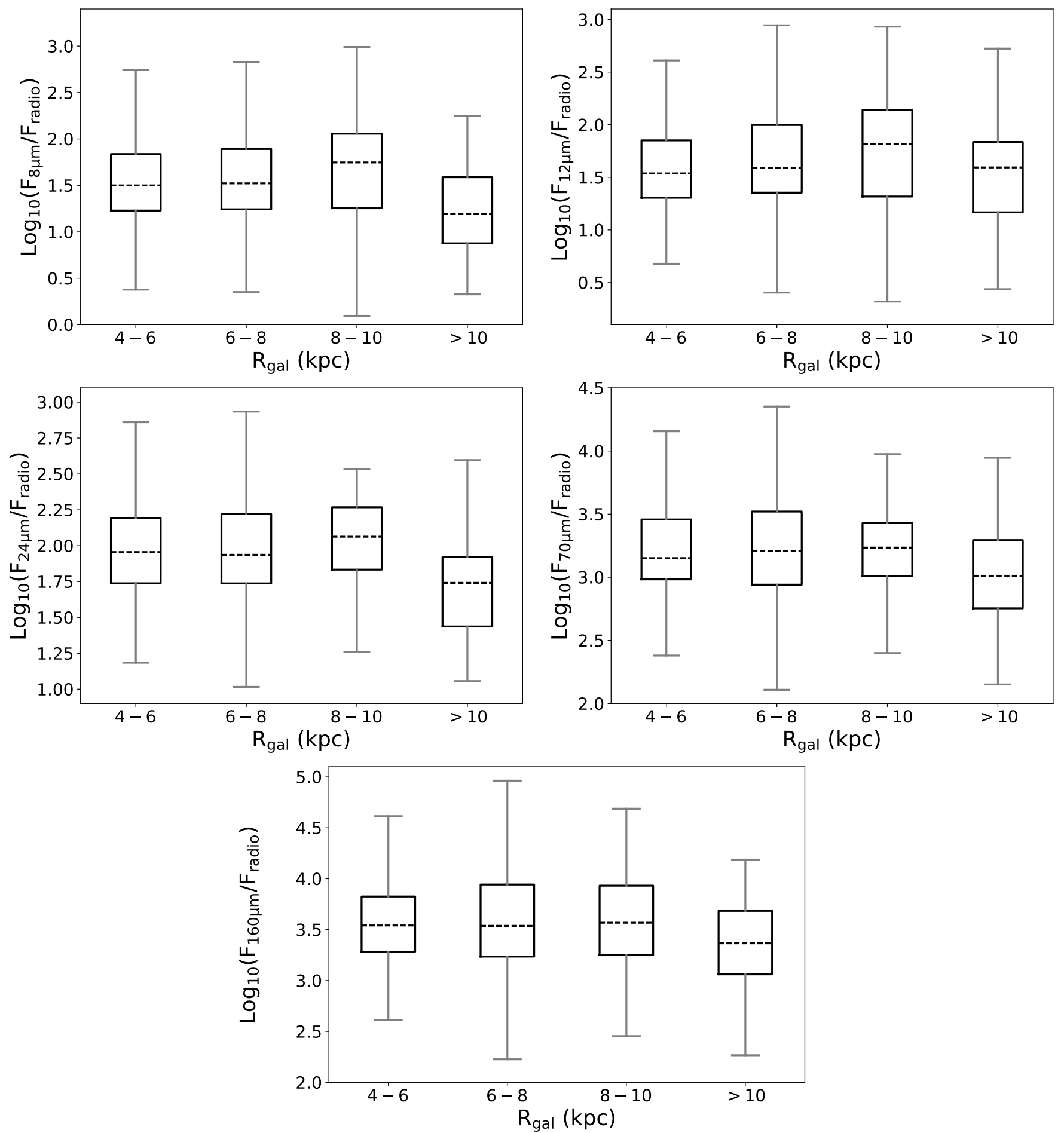

Figure 11. Box and whiskers plots as in Figure 7 for the IR/radio flux density ratios in four Galactocentric radii bins. The plots, show no trend in IR/radio ratios for H II regions with $R_{\text {gal }}$, although the regions with $R_{\text {gal }}>10 \mathrm{kpc}$ do show consistently lower ratios. The label $F_{\text {radio }}$ denotes the combination of the $20 \mathrm{~cm}$ and the $21 \mathrm{~cm}$ radio continuum data (see Section 4.3 ). 

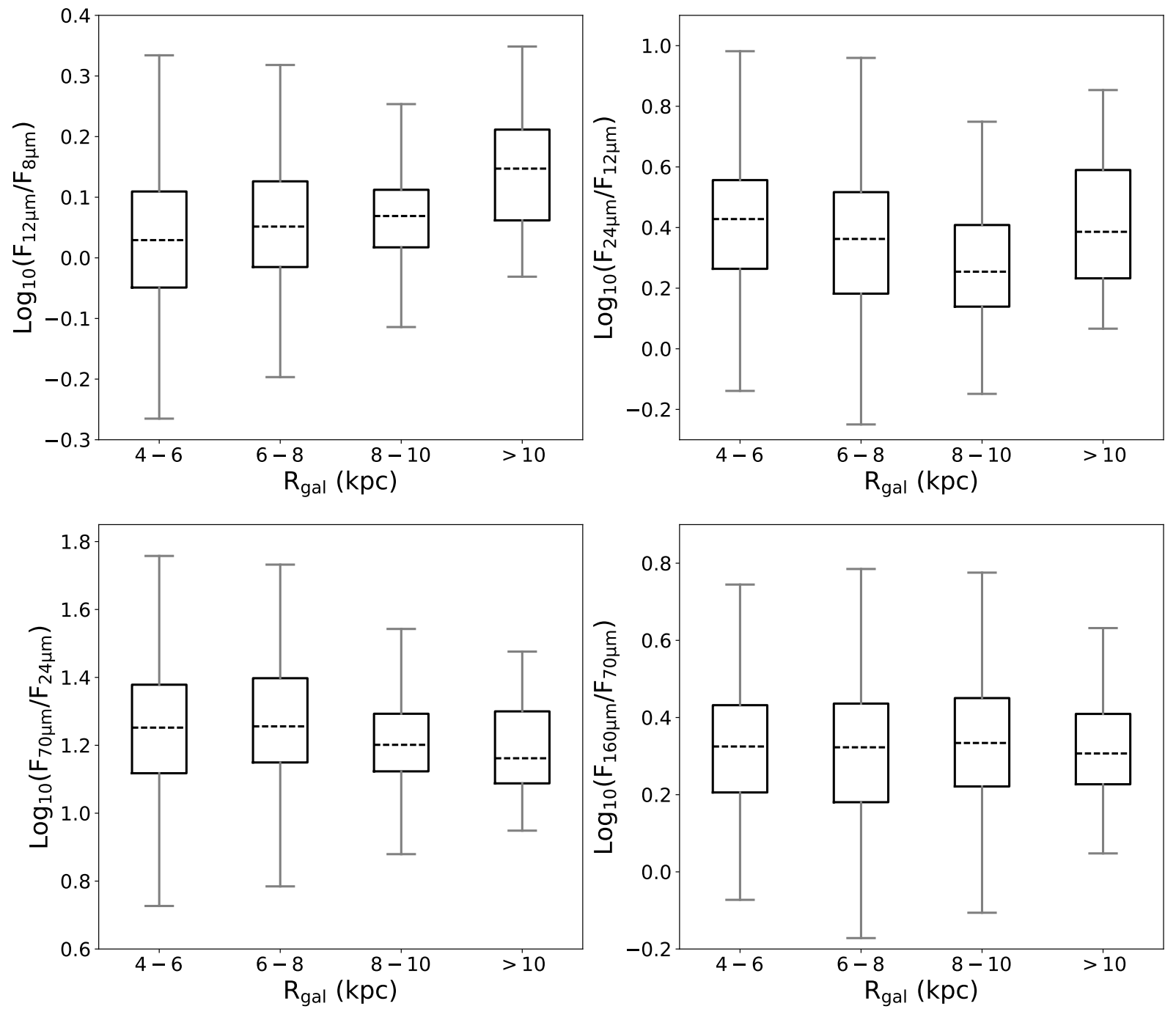

Figure 12. Box and whiskers plots as in Figure 7 for IR colors in four Galactocentric radii bins. The plots, show similar infrared colors for all H II regions, regardless of $R_{\text {gal }}$, except the $\log _{10}\left(F_{12 \mu \mathrm{m}} / F_{8} \mu \mathrm{m}\right)$ ratio which shows slightly increasing trend, and the $\log _{10}\left(F_{24 \mu \mathrm{m}} / F_{12 \mu \mathrm{m}}\right)$ ratio that shows some fluctuations. 
Table 4. The median IR to radio, and IR flux density ratios as a function of $R_{g a l}$

\begin{tabular}{|c|c|c|c|c|c|}
\hline Flux density ratios & $\begin{array}{l}\mathrm{R}_{\text {gal }} \\
{[\mathrm{kpc}]}\end{array}$ & Median & $\sigma$ & $\begin{array}{c}\text { Color criteria } \\
(\mathrm{Q} 1-1.5 \times \mathrm{IQR}-\mathrm{Q} 3+1.5 \times \mathrm{IQR})^{*}\end{array}$ & \# of sources \\
\hline \multirow{5}{*}{$\log _{10}\left(\mathrm{~F}_{8 \mu \mathrm{m}} / \mathrm{F}_{\text {radio }}\right)$} & $4<R_{g a l}<6$ & 1.50 & 0.34 & $0.38-2.75$ & 322 \\
\hline & $6<R_{\text {gal }}<8$ & 1.52 & 0.37 & $0.35-2.83$ & 260 \\
\hline & $8<R_{\text {gal }}<10$ & 1.75 & 0.50 & $0.10-2.99$ & 48 \\
\hline & $R_{\text {gal }}>10$ & 1.20 & 0.32 & $0.33-2.25$ & 41 \\
\hline & All & 1.43 & 0.38 & $0.10-2.99$ & 658 \\
\hline \multirow{5}{*}{$\log _{10}\left(F_{12 \mu \mathrm{m}} / \mathrm{F}_{\text {radio }}\right)$} & $4<R_{g a l}<6$ & 1.54 & 0.29 & $0.68-2.61$ & 327 \\
\hline & $6<R_{\text {gal }}<8$ & 1.59 & 0.41 & $0.41-2.94$ & 264 \\
\hline & $8<R_{\text {gal }}<10$ & 1.82 & 0.50 & $0.32-2.93$ & 46 \\
\hline & $R_{\text {gal }}>10$ & 1.59 & 0.25 & $0.44-2.72$ & 41 \\
\hline & All & 1.64 & 0.36 & $0.32-2.94$ & 678 \\
\hline \multirow{5}{*}{$\log _{10}\left(\mathrm{~F}_{24 \mu \mathrm{m}} / \mathrm{F}_{\text {radio }}\right)$} & $4<R_{g a l}<6$ & 1.96 & 0.23 & $1.18-2.86$ & 324 \\
\hline & $6<R_{\text {gal }}<8$ & 1.94 & 0.28 & $1.02-2.93$ & 259 \\
\hline & $8<R_{\text {gal }}<10$ & 2.06 & 0.23 & $1.26-2.53$ & 45 \\
\hline & $R_{g a l}>10$ & 1.74 & 0.30 & $1.06-2.60$ & 25 \\
\hline & All & 1.93 & 0.26 & $1.02-2.93$ & 653 \\
\hline \multirow{5}{*}{$\log _{10}\left(F_{70 \mu \mathrm{m}} / \mathrm{F}_{\text {radio }}\right)$} & $4<R_{\text {gal }}<6$ & 3.15 & 0.31 & $2.38-4.16$ & 330 \\
\hline & $6<R_{\text {gal }}<8$ & 3.21 & 0.28 & $2.11-4.35$ & 268 \\
\hline & $8<R_{\text {gal }}<10$ & 3.23 & 0.25 & $2.40-3.98$ & 46 \\
\hline & $R_{g a l}>10$ & 3.01 & 0.32 & $2.15-3.95$ & 33 \\
\hline & All & 3.15 & 0.29 & $2.11-4.16$ & 677 \\
\hline \multirow{5}{*}{$\log _{10}\left(F_{160 \mu \mathrm{m}} / \mathrm{F}_{\text {radio }}\right)$} & $4<R_{g a l}<6$ & 3.54 & 0.29 & $2.61-4.61$ & 326 \\
\hline & $7<R_{\text {gal }}<8$ & 3.54 & 0.40 & $2.23-4.96$ & 261 \\
\hline & $8<R_{\text {gal }}<10$ & 3.57 & 0.37 & $2.45-4.69$ & 46 \\
\hline & $R_{\text {gal }}>10$ & 3.37 & 0.29 & $2.27-4.19$ & 33 \\
\hline & All & 3.51 & 0.34 & $2.23-4.96$ & 665 \\
\hline \multirow{5}{*}{$\log _{10}\left(\mathrm{~F}_{12 \mu \mathrm{m}} / \mathrm{F}_{8 \mu \mathrm{m}}\right)$} & $4<R_{g a l}<6$ & 0.03 & 0.09 & $-0.27-0.33$ & 327 \\
\hline & $6<R_{\text {gal }}<8$ & 0.05 & 0.08 & $-0.20-0.32$ & 306 \\
\hline & $8<R_{\text {gal }}<10$ & 0.07 & 0.05 & $-0.11-0.25$ & 56 \\
\hline & $R_{\text {gal }}>10$ & 0.15 & 0.09 & $-0.03-0.35$ & 30 \\
\hline & All & 0.08 & 0.08 & $-0.27-0.35$ & 719 \\
\hline \multirow{5}{*}{$\log _{10}\left(\mathrm{~F}_{24 \mu \mathrm{m}} / \mathrm{F}_{12 \mu \mathrm{m}}\right)$} & $4<R_{\text {gal }}<6$ & 0.43 & 0.17 & $-0.14-0.98$ & 328 \\
\hline & $6<R_{\text {gal }}<8$ & 0.36 & 0.18 & $-0.25-0.96$ & 304 \\
\hline & $8<R_{\text {gal }}<10$ & 0.25 & 0.16 & $-0.15-0.75$ & 55 \\
\hline & $R_{\text {gal }}>10$ & 0.39 & 0.20 & $0.07-0.85$ & 27 \\
\hline & All & 0.36 & 0.18 & $-0.25-0.98$ & 714 \\
\hline
\end{tabular}


Table 4 (continued)

\begin{tabular}{cccccc}
\hline \hline Flux density ratios & $\begin{array}{c}\mathrm{R}_{\text {gal }} \\
{[\mathrm{kpc}]}\end{array}$ & Median & $\sigma$ & $\begin{array}{c}\text { Color criteria } \\
(\mathrm{Q} 1-1.5 \times \mathrm{IQR}-\mathrm{Q} 3+1.5 \times \mathrm{IQR})^{*}\end{array}$ & \# of sources \\
& & & & & \\
& $4<R_{\text {gal }}<6$ & 1.25 & 0.13 & $0.73-1.76$ & 331 \\
& $6<R_{\text {gal }}<8$ & 1.26 & 0.14 & $0.78-1.73$ & 306 \\
$\log _{10}\left(\mathrm{~F}_{70 \mu \mathrm{m}} / \mathrm{F}_{24 \mu \mathrm{m}}\right)$ & $8<R_{\text {gal }}<10$ & 1.20 & 0.09 & $0.88-1.54$ & 54 \\
& $R_{\text {gal }}>10$ & 1.16 & 0.14 & $0.95-1.48$ & 718 \\
& All & 1.22 & 0.13 & $0.73-1.76$ & 332 \\
& $4<R_{\text {gal }}<6$ & 0.32 & 0.11 & $-0.07-0.74$ & 313 \\
& $6<R_{\text {gal }}<8$ & 0.32 & 0.14 & $-0.17-0.79$ & 36 \\
$\log _{10}\left(\mathrm{~F}_{160 \mu \mathrm{m}} / \mathrm{F}_{70 \mu \mathrm{m}}\right)$ & $8<R_{\text {gal }}<10$ & 0.33 & 0.11 & $-0.11-0.78$ & 736 \\
& $R_{\text {gal }}>10$ & 0.31 & 0.10 & $0.05-0.63$ & $-0.17-0.79$ \\
\hline All & 0.32 & 0.11 & & \\
\hline
\end{tabular}

${ }^{*} Q 1$ and $Q 3$ are the $25^{t} h$ and $75^{t} h$ percentile of the data, respectively, and $I Q R=(Q 3-Q 1)$ is the interquartile.

a fuction of Galactocentric radius, $R_{\text {gal }}$. We summarize the results of these two Figures in Table 4, and graphically in Figure 13.

Interestingly, the IR to radio ratios do not show a consistent trend with $R_{\text {gal }}$ (Figure 11); H II regions throughout the Galaxy have similar IR to radio ratios. Again, the final bin $R_{\text {gal }}>10 \mathrm{kpc}$ shows some deviation from this trend compared with the rest of the Galaxy. The radio continuum is a proxy for the power of the ionizing source(s), where as the IR arises from various dust species associated with the regions. A lower IR to radio ratio in the outer Galaxy may be caused by a lack of dust, a lack of photons to excite the dust, or both.

Crocker et al. (2013) investigated the ratio of $8 \mu \mathrm{m}$ to $\mathrm{H} \alpha$ for $\mathrm{H}$ II regions in NGC628 as a function of galactocentric radius. They found that relative to $\mathrm{H} \alpha$, the $8 \mu \mathrm{m}$ emission decreases with increasing galactocentric radius. Over the range of galactocentric radii probed, the $\mathrm{H} \alpha$ to $8 \mu \mathrm{m}$ flux density ratio decreases by a factor of 4.0. Crocker et al. (2013) suggested that at high $R_{\text {gal }}$ there are fewer photons absorbed by PAHs, leading to lower $8 \mu \mathrm{m}$ emission. They also suggested that the negative trend they found may be caused by the metallicity gradient in the disk, because $\mathrm{PAH}$ emission is weaker at lower metallicities (e.g. Engelbracht et al. 2005; Galliano et al. 2005).

Although we do not have $\mathrm{H} \alpha$ data for all regions, radio continuum traces the same ionized gas. We fit a linear regression line to the $\log _{10}\left(F_{8 \mu \mathrm{m}} / F_{\text {radio }}\right)$ ratio. We find a slight negative trend, in agreement with Crocker et al.
(2013). The slope of the fit, however, $-0.006 \pm 0.009$, is flat within the uncertainties over the range of $R_{\text {gal }}$ probed here $(\sim 4-15 \mathrm{kpc})$. Only $\sim 7 \%$ of the the data points are located in the outer Galaxy $\left(R_{G}>8.5 \mathrm{kpc}\right)$, and nearly all (80\%) of the outer Galaxy data points fall below the fit line. Since the fit can be biased by the low number of data points in the outer Galaxy, we also fit the $500 \mathrm{pc}$ binned data (filled triangles on Figure 14). This fit also shows a weak negaive trend. Additional outer Galaxy data points would help to determine the strength of the relationship.

\section{SUMMARY}

We derived the infrared $(8-160 \mu \mathrm{m})$ and radio $(20 \mathrm{~cm}$ and $21 \mathrm{~cm}$ ) flux densities for $\sim 1000 \mathrm{H}$ II regions from the WISE Catalog of Galactic H II Regions to investigate relationships between the flux densities, angular sizes, and Galactocentric radii. The investigated H II regions are located in the first Galactic quadrant $(17.5<\ell<$ $\left.65^{\circ}\right)$ where kinematic distances are relatively accurate.

Comparing VGPS and MAGPIS flux densities, we were unable to reproduce the result of Helfand et al. (2006) that MAGPIS is overestimating the flux densities of large sources. This indicates that either MAGPIS is well-calibrated, or that both the VGPS and MAGPIS underestimate the flux densities of large sources. By comparing $21 \mathrm{~cm}$ flux densities with those derived at $3 \mathrm{~cm}$, we showed that radio optical depth effects are statistically unimportant for our sample.

All measured IR and radio flux densities are highly correlated, with a higher scatter at the lower end of the 

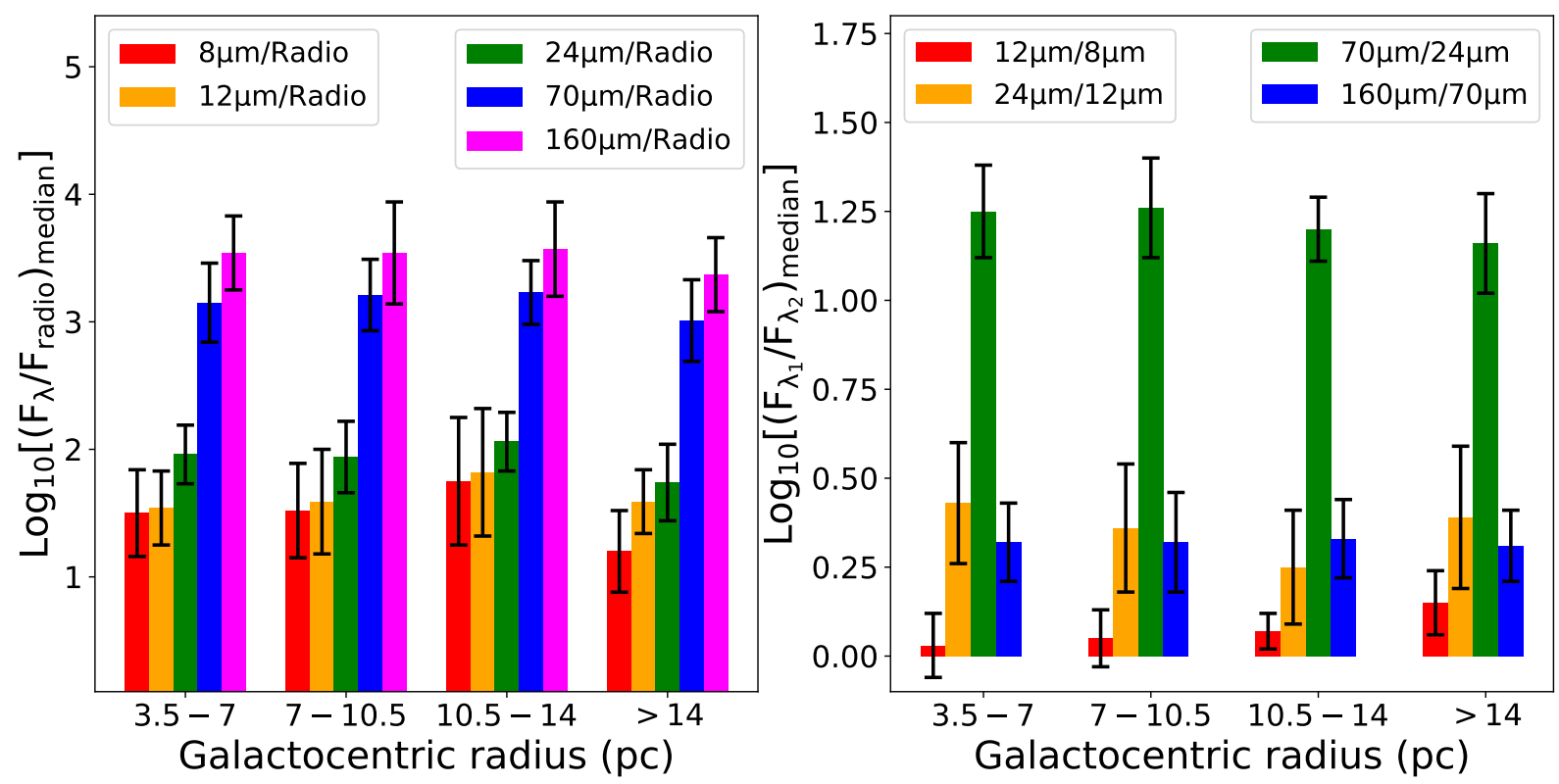

Figure 13. Graphical representation of the median values within the $R_{\text {gal }}$ bins from Table 4 . The standard deviation of all data points are marked with the error bars. The term "radio" means the combination of the $20 \mathrm{~cm}$ and the $21 \mathrm{~cm}$ radio continuum data, see Section 4.3. On the left plot, we show the ratios of the IR and radio flux densities. There is essentially no change in the IR to radio flux density ratios with $R_{\text {gal }}$, altough the $8 \mu \mathrm{m}$ to radio ratio is low for the regions with the largest $R_{\text {gal }}$ values. The right plot show the distribution of median values of color indices. This plot suggests that all the investigated IR flux density ratios are unchanged within the errors, independent of Galactocentric radius.

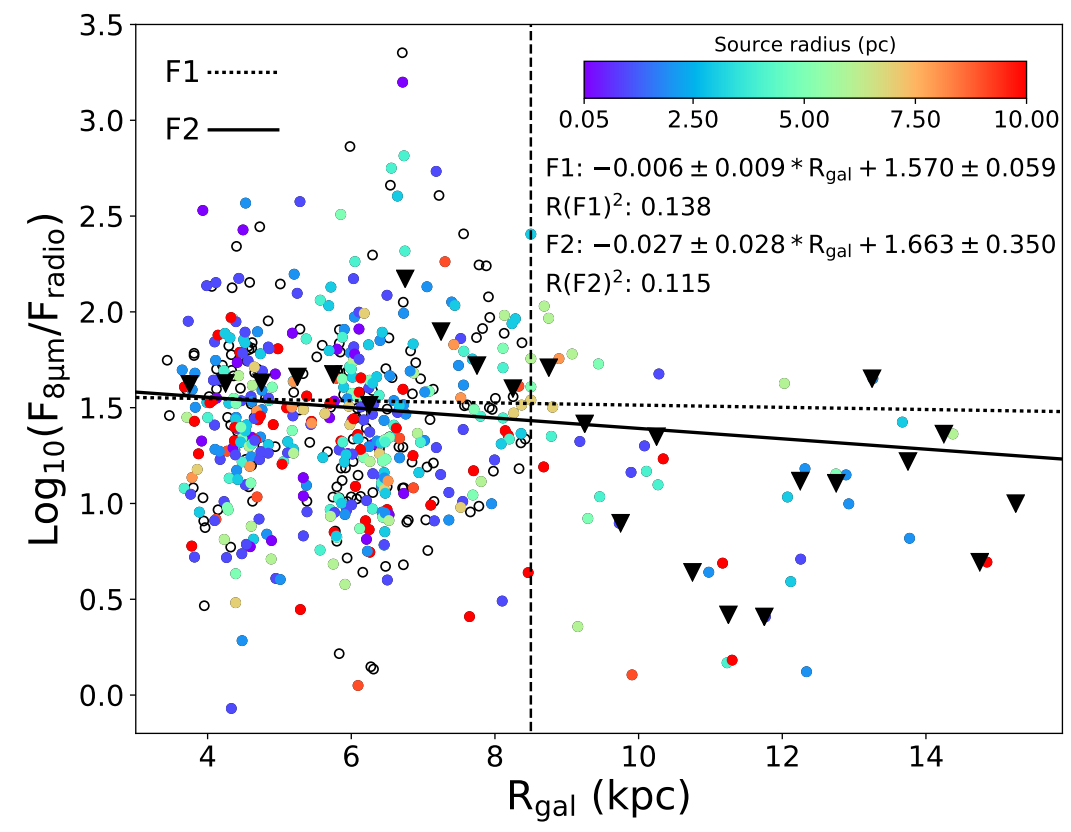

Figure 14. The $\log _{10}\left(F_{8 \mu \mathrm{m}} / F_{\text {radio }}\right)$ flux density ratio of $\sim 600 \mathrm{H}$ II regions as a function of Galactocentric radius. The $F_{\text {radio }}$ denotes the combination of the $20 \mathrm{~cm}$ and the $21 \mathrm{~cm}$ radio continuum data, see Section 4.3 . The empty circles mark the sources that lack Heliocentric distances. The vertical dashed line denotes the boundary between the inner and outer Galaxy (at $8.5 \mathrm{kpc}$ ), the dotted line shows the best fit to all data points and the solid line is the best fit to the $250 \mathrm{pc}$ binned data (filled triangles). The fits are similar within the fit uncertainties. 
flux densities. With the exception of $70 \mu \mathrm{m}$ data, the IR emissions have similar strong correlation with radio data. Removing the smallest regions from the fit increases the correlation between the IR and radio flux densities.

All H II regions have similar infrared flux density ratios, regardless of H II region physical size. WC89 suggested that ultra-compact (UC) H II regions alone are well separated from other sources found in the IRAS Point Source Catalog. Our results show that HII regions of all physical sizes can satisfy their criteria, not just UC HII regions, in agreement with previous studies (e.g. Anderson et al. 2012; Leto et al. 2009; Chini et al. 1987). This result implies that the total number of UC H II regions in the Galaxy as derived from IR color indices is significantly uncertain. The 160 to $70 \mu \mathrm{m}$ flux density ratio is above unity for nearly all regions, which implies that emission from large, cold dust grains dominates the spectral energy distributions. Using our data, we suggest that the $\log _{10}\left(F_{24 \mu \mathrm{m}} / F_{12 \mu \mathrm{m}}\right) \geq 0$ and $\log _{10}\left(F_{70 \mu \mathrm{m}} / F_{12 \mu \mathrm{m}}\right) \geq 1.2$, and $\log _{10}\left(F_{24 \mu \mathrm{m}} / F_{12 \mu \mathrm{m}}\right) \geq$ 0 and $\log _{10}\left(F_{160 \mu \mathrm{m}} / F_{70 \mu \mathrm{m}}\right) \leq 0.67$ IR color indice pairs can be used as an approximate IR limits for H II regions, independent of size.

We find a trend of weakly decreasing IR to radio flux density ratios with increasing $R_{\text {gal }}$. This has been noted for external galaxies (Crocker et al. 2013), and we confirm their results using $8.0 \mu \mathrm{m}$ and radio continuum data. Since the IR emission traces dust, and the trend is seen in all IR wavelengths, this result indicates that there may be decreasing dust abundance in the outer Galaxy.

We thank the anonymous referee for his/her comments, which improved the paper. This work was supported by NASA ADAP grant NNX12AI59G to LDA and NSF grant AST1516021 to LDA. PACS has been developed by a consortium of institutes led by MPE (Germany) and including UVIE (Austria); KU Leuven, CSL, IMEC (Belgium); CEA, LAM (France); MPIA (Germany); INAF-IFSI/OAA/OAP/OAT, LENS, SISSA (Italy); IAC (Spain). This development has been supported by the funding agencies BMVIT (Austria), ESAPRODEX (Belgium), CEA/CNES (France), DLR (Germany), ASI/INAF (Italy), and CICYT/MCYT (Spain). This research has made use of NASAs Astrophysics Data System Bibliographic Services and the SIMBAD database operated at CDS, Strasbourg, France. This publication makes use of data products from WISE, which is a joint project of the University of California, Los Angeles, and the Jet Propulsion Laboratory/California Institute of Technology, funded by the National Aeronautics and Space Administration. This research has made use of NASA's Astrophysics Data System Bibliographic Services.

\section{REFERENCES}

Anderson, L. D., Bania, T. M., Balser, D. S., et al. 2014, ApJS, 212,1

Anderson, L. D., Bania, T. M., Balser, D. S., \& Rood, R. T. 2011, ApJS, 194, 32

Anderson, L. D., Zavagno, A., Barlow, M. J., García-Lario, P., \& Noriega-Crespo, A. 2012, A\&A, 537, A1

Andrews, H., Boersma, C., Werner, M. W., et al. 2015, ApJ, 807, 99

Bania, T. M., Anderson, L. D., Balser, D. S., \& Rood, R. T. 2010, ApJL, 718, L106

Becker, R. H., White, R. L., Helfand, D. J., \& Zoonematkermani, S. 1994, ApJS, 91, 347

Benjamin, R. A., Churchwell, E., Babler, B. L., et al. 2003, PASP, 115, 953

Boggs, P. T., \& Rogers, J. E. 1990, Contemporary Mathematics, 112,183

Broadbent, A., Osborne, J. L., \& Haslam, C. G. T. 1989, MNRAS, 237, 381

Calzetti, D., Wu, S.-Y., Hong, S., et al. 2010, ApJ, 714, 1256

Carey, S. J., Noriega-Crespo, A., Price, S. D., et al. 2005, in Bulletin of the American Astronomical Society, Vol. 37, American Astronomical Society Meeting Abstracts, 1252

Chini, R., Kruegel, E., \& Wargau, W. 1987, A\&A, 181, 378

Churchwell, E. 2002, ARA\&A, 40, 27

Churchwell, E., \& GLIMPSE Team. 2001, in Bulletin of the American Astronomical Society, Vol. 33, American Astronomical Society Meeting Abstracts \#198, 821

Cohen, M. 2009, AJ, 137, 3449
Cohen, M., Green, A. J., Meade, M. R., et al. 2007, MNRAS, 374,979

Crocker, A. F., Calzetti, D., Thilker, D. A., et al. 2013, ApJ, 762,79

de Jong, T., Klein, U., Wielebinski, R., \& Wunderlich, E. 1985, A\&A, 147, L6

De Pree, C. G., Peters, T., Mac Low, M.-M., et al. 2014, ApJL, 781, L36

Deharveng, L., Schuller, F., Anderson, L. D., et al. 2010, A\&A, 523, A6

Dopita, M. A., Fischera, J., Crowley, O., et al. 2006, ApJ, 639, 788

Dreher, J. W., \& Welch, W. J. 1981, ApJ, 245, 857

Engelbracht, C. W., Gordon, K. D., Rieke, G. H., et al. 2005, ApJL, 628, L29

Fazio, G. G., Hora, J. L., Willner, S. P., et al. 1998, in Proc. SPIE, Vol. 3354, Infrared Astronomical Instrumentation, ed. A. M. Fowler, 1024-1031

Galliano, F., Madden, S. C., Jones, A. P., Wilson, C. D., \& Bernard, J.-P. 2005, A\&A, 434, 867

Green, D. A. 2004, Bulletin of the Astronomical Society of India, 32,335

Griffin, M. J., Abergel, A., Abreu, A., et al. 2010, A\&A, 518, L3

Haddock, F. T., Mayer, C. H., \& Sloanaker, R. M. 1954, ApJ, 119,456

Harper, D. A. 1974, ApJ, 192, 557

Haslam, C. G. T., \& Osborne, J. L. 1987, Nature, 327, 211 
Helfand, D. J., Becker, R. H., White, R. L., Fallon, A., \& Tuttle, S. 2006, AJ, 131, 2525

Helfand, D. J., Zoonematkermani, S., Becker, R. H., \& White, R. L. 1992, ApJS, 80, 211

Helou, G. 1986, ApJL, 311, L33

Hughes, V. A., \& MacLeod, G. C. 1989, AJ, 97, 786

Kim, K.-T., \& Koo, B.-C. 2002, ApJ, 575, 327

Leto, P., Umana, G., Trigilio, C., et al. 2009, A\&A, 507, 1467

Low, F. J., \& Aumann, H. H. 1970, ApJL, 162, L79

Mingo, B., Watson, M. G., Rosen, S. R., et al. 2016, MNRAS, 462,2631

Molinari, S., Swinyard, B., Bally, J., et al. 2010, PASP, 122, 314

Molinari, S., Schisano, E., Elia, D., et al. 2016, A\&A, 591, A149

Paladini, R., Umana, G., Veneziani, M., et al. 2012, ApJ, 760, 149

Peters, T., Banerjee, R., Klessen, R. S., et al. 2010a, ApJ, 711, 1017

Peters, T., Mac Low, M.-M., Banerjee, R., Klessen, R. S., \& Dullemond, C. P. 2010b, ApJ, 719, 831

Phillips, J. P., \& Ramos-Larios, G. 2008, MNRAS, 391, 1527

Piddington, J. H. 1951, MNRAS, 111, 45

Pilbratt, G. L., Riedinger, J. R., Passvogel, T., et al. 2010, A\&A, 518, L1

Poglitsch, A., Waelkens, C., Geis, N., et al. 2010, A\&A, 518, L2

Reich, P., \& Reich, W. 1986, A\&AS, 63, 205

Reich, W., Reich, P., \& Fuerst, E. 1990, A\&AS, 83, 539
Rieke, G. H., Young, E. T., Engelbracht, C. W., et al. 2004, ApJS, 154, 25

Robitaille, T. P., Churchwell, E., Benjamin, R. A., et al. 2012, A\&A, 545, A39

Roser, J. E., \& Ricca, A. 2015, ApJ, 801, 108

Sanders, D. B., Mazzarella, J. M., Kim, D.-C., Surace, J. A., \& Soifer, B. T. 2003, AJ, 126, 1607

Siebenmorgen, R., \& Krügel, E. 2007, A\&A, 461, 445

Soifer, B. T., \& Neugebauer, G. 1991, AJ, 101, 354

Spitzer, L. 1978, Physical processes in the interstellar medium, doi:10.1002/9783527617722

Stil, J. M., Taylor, A. R., Dickey, J. M., et al. 2006, AJ, 132, 1158

Strömgren, B. 1939, ApJ, 89, 526

Tabatabaei, F. S., Berkhuijsen, E. M., Frick, P., Beck, R., \& Schinnerer, E. 2013, A\&A, 557, A129

Temi, P., Brighenti, F., \& Mathews, W. G. 2009, ApJ, 707, 890

Tielens, A. G. G. M. 2008, ARA\&A, 46, 289

Wang, G. 1991, Acta Astrophysica Sinica, 11, 311

Westerhout, G. 1958, BAN, 14, 215

White, R. L., Becker, R. H., \& Helfand, D. J. 1991, ApJ, 371, 148

Wood, D. O. S., \& Churchwell, E. 1989a, ApJ, 340, 265

-. 1989b, ApJS, 69, 831

Wright, E. L., Eisenhardt, P. R. M., Mainzer, A. K., et al. 2010, AJ, 140, 1868

Zoonematkermani, S., Helfand, D. J., Becker, R. H., White, R. L., \& Perley, R. A. 1990, ApJS, 74, 181 


\section{APPENDIX}

\section{A. WISE CATALOG WEB SITE}

We have updated the WISE Catalog Web Site ${ }^{9}$ with the aperture photometry results given here.

\section{B. FLUX DENSITY UNCERTAINTIES}

To check the uncertainties of the derived IR $(8 \mu \mathrm{m}, 12 \mu \mathrm{m}, 22 \mu \mathrm{m}, 24 \mu \mathrm{m}, 70 \mu \mathrm{m}$, and $160 \mu \mathrm{m}$,$) and radio (20 \mathrm{~cm}$ and $21 \mathrm{~cm}$ ) flux densities, we investigate their relative (or fractional) errors (Figures B1 and B2). The number distributions of the fractional errors ( B1) show that, considering all wavelengths, $50 \%$ of the data lies under $\sim 35 \%$, while $90 \%$ of the data is under $\sim 191 \%$ of fractional uncertainty. We also investigated the relative errors as a function of flux density values, Figure B2. These plots suggest that the fractional errors are not correlated with their flux density values, independent of wavelengths.

Table B1. Fractional error limits at $50 \%$ and $90 \%$ of the data points.

\begin{tabular}{|c|c|c|}
\hline \multirow[t]{2}{*}{$\lambda$} & \multicolumn{2}{|c|}{$\sigma F_{\lambda} / F_{\lambda}(\%)$} \\
\hline & at $50 \%$ of data & at $90 \%$ of data \\
\hline $8 \mu \mathrm{m}$ & $\sim 26 \%$ & $\sim 126 \%$ \\
\hline $12 \mu \mathrm{m}$ & $\sim 29 \%$ & $\sim 132 \%$ \\
\hline $22 \mu \mathrm{m}$ & $\sim 20 \%$ & $\sim 102 \%$ \\
\hline $24 \mu \mathrm{m}$ & $\sim 18 \%$ & $\sim 79 \%$ \\
\hline $70 \mu \mathrm{m}$ & $\sim 17 \%$ & $\sim 86 \%$ \\
\hline $160 \mu \mathrm{m}$ & $\sim 35 \%$ & $\sim 191 \%$ \\
\hline $20 \mathrm{~cm}$ & $\sim 13 \%$ & $\sim 49 \%$ \\
\hline $21 \mathrm{~cm}$ & $\sim 30 \%$ & $\sim 92 \%$ \\
\hline
\end{tabular}

9 http://astro.phys.wvu.edu/wise/ 

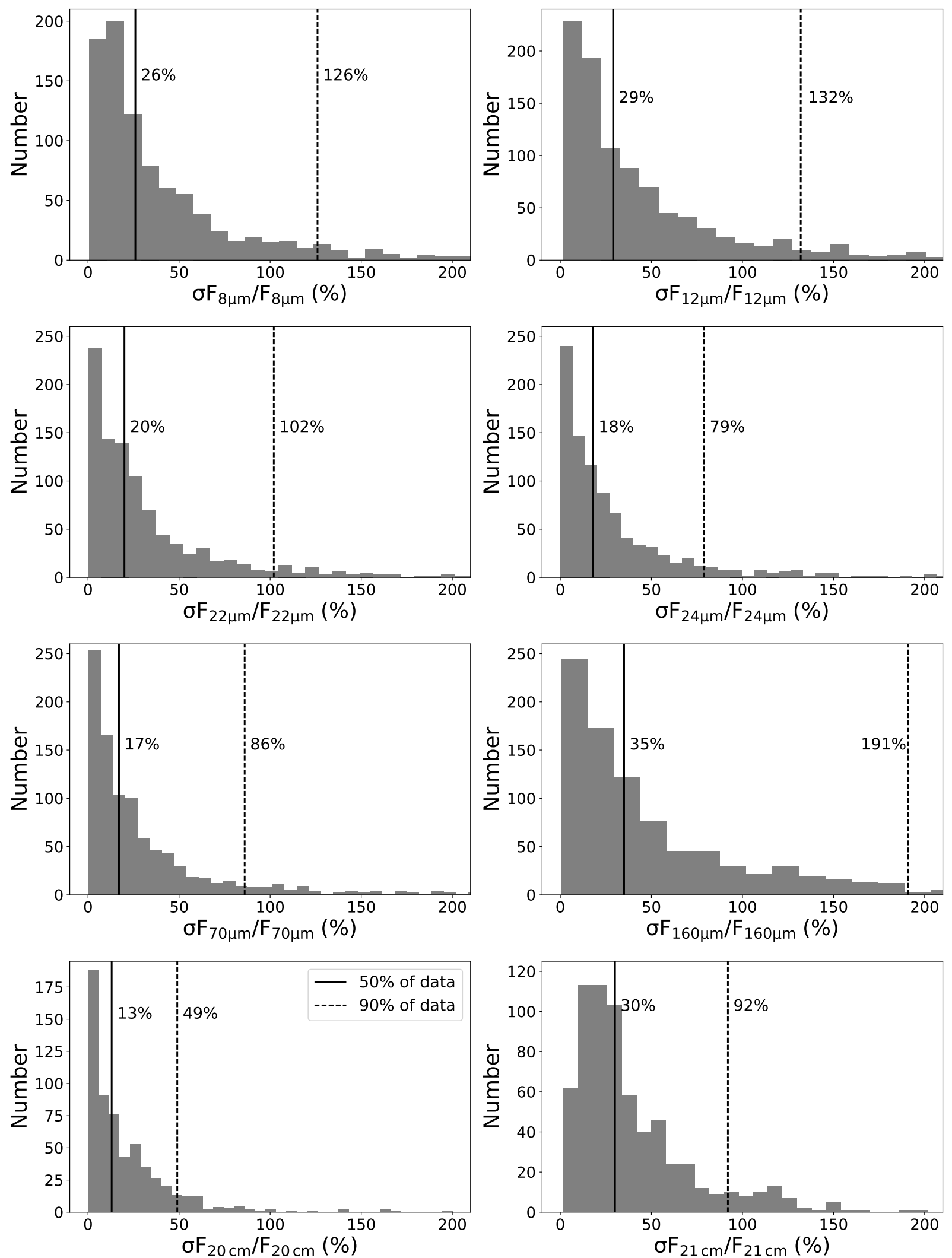

Figure B1. Distribution of fractional uncertainties $\left(\sigma F_{\lambda} / F_{\lambda}\right)$. The solid and dashed lines represent the fractional error limits at a given wavelength, for the $50 \%$ and $90 \%$ of the data, respectively (marked in the lower left subplot). 

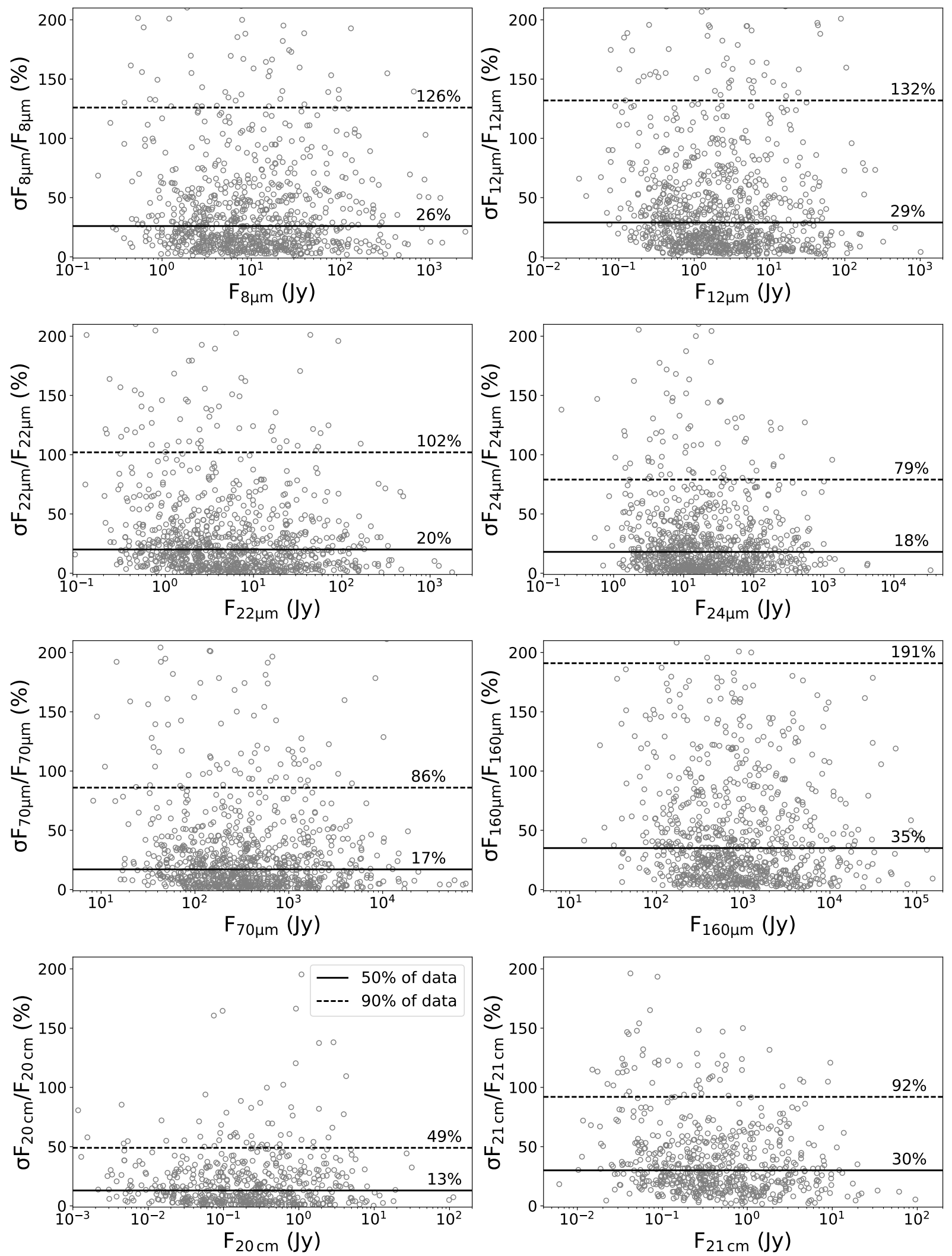

Figure B2. Distribution of fractional uncertainties as a function of flux density. Horizontal lines represent the same as in Figure B1. The fractional uncertainties are not strongly correlated with the flux densities. 